\title{
Block-iterative Richardson-Lucy methods for image deblurring
}

\author{
Nam-Yong Lee
}

\begin{abstract}
In this paper, we extend the Richardson-Lucy (RL) method to block-iterative versions, separated BI-RL, and interlaced BI-RL, for image deblurring applications. We propose combining algorithms for separated BI-RL to form block artifact-free output images from separately deblurred block images. For interlaced BI-RL to accelerate the iteration, we propose an interlaced block-iteration algorithm on down-sampled blocks of the observed image. Simulation studies show that separated BI-RL and interlaced BI-RL achieve desired goals in Gaussian and diagonal deblurrings.
\end{abstract}

Keywords: Parallel computation; Block artifacts; Ordered subsets; Accelerated iteration

\section{Introduction}

The image deblurring problem has many applications in science and engineering fields, and many methods have been proposed for it [1]. Among them, the RichardsonLucy (RL) method, which was proposed independently by Richardson [2] and Lucy [3] in 1970s, has been one of the most widely used iterative deblurring methods. Applications of RL in microscopy, astronomy, or motion deblurring can be found in $[4,5]$ and references therein. Variants of RL with specific purposes such as adaptivity, parallel implementation, acceleration, suppression of ringing/boundary artifacts, or edge-preserving can be found in [6-12].

In the paper by Shepp and Vardi [13], the same algorithm was re-derived from the maximization likelihood principle in the application to emission tomography and the expectation maximization (EM) method in [14]. In the paper by Hudson and Larkin [6], the ordered subsets expectation maximization (OSEM) method was proposed as an acceleration technique for EM. Since its introduction, OSEM has been successfully used for many applications in emission tomography $[15,16]$.

The use of RL in image deblurring is, however, computer intensive and often suffers from slow convergence. To deal with these obstacles, block-iterative RL (BI-RL) methods have been proposed $[17,18]$. BI-RL decomposes the main problem into several sub-problems by grouping pixels of

Correspondence: nylee@inje.ac.kr

Department of Applied Mathematics, Inje University, Kimhae, Gyeongnam, Korea the observed image into several subsets and applies RL to each sub-problem. In this paper, image parts defined on those grouped pixel subsets will be called blocks. In [17], the observed image was decomposed to four rectangular blocks, RL was applied to each block separately, and the final image was obtained by combining four deblurred block images. In [18], an OSEM-like method was proposed to accelerate the iteration by using multi-views of the image. The term 'multi-views of the image' means that the image to be recovered is observed several times under different imaging environments. The observed data at each imaging environment is often called a view of the image. The problem of deblurring images from multiviews is also called multiple image deconvolution [4].

In this paper, we extend methods in $[17,18]$ to separated BI-RL and interlaced BI-RL, for image deblurring applications.

The goal of separated BI-RL is to combine separately deblurred sub-image blocks into the resulting output image without block artifacts. Obviously, this is not a new approach. What is new in the proposed separated BI-RL is that the proposed method can suppress block artifacts efficiently for arbitrarily shaped blocks. This flexibility is important for the success of separated BI-RL; simulation results of this paper will show that diagonally shaped blocks produce better results than rectangularly shaped blocks for separated BI-RL in the deblurring problem modeled by a diagonal point spread function (PSF).

\section{Springer}


The goal of interlaced BI-RL is the accelerated iteration by using an OSEM-like method as in [18]. Image deblurring problems of this paper, however, are assumed to have only one view of the image, unlike in [18]. To overcome this 'one view' limitation, the proposed interlaced BI-RL decomposes the observed image into several downsampled blocks and treats those down-sampled blocks as multi-views of the image. Interlaced BI-RL accelerates the iteration by using the one-step RL block iterates as the starting image in the next block iteration, as OSEM does in emission tomography.

The performance of proposed methods depends on how blocks are formed. There are many possibilities for the block partition. It is, however, not clear how blocks should be formed for a specific PSF.

The work in this paper will be restricted to the test of rectangular or diagonal sub-image blocks for separated BI-RL and rectangularly- or diagonally-down-sampled blocks for interlaced BI-RL in Gaussian and diagonal deblurrings. While explaining test results, possible extensions of proposed methods to general deblurring problems will be presented briefly.

\section{Definition and background}

\subsection{Image deblurring problem}

We assume that the true image $\mathbf{f}$ is defined on an index set $\Omega$ and its observed version $\mathbf{g}$ on an index set $\Lambda$. We also assume that $\mathbf{g}$ is blurred from $\mathbf{f}$ by a linear operator $\mathcal{T}: \ell^{2}(\Omega) \rightarrow \ell^{2}(\Lambda)$ and further corrupted by pixel-wisely independent Poisson noise:

$$
g_{i_{1}, i_{2}} \sim \operatorname{Poisson}\left((\mathcal{T} \mathbf{f})_{i_{1}, i_{2}}\right), \quad\left(i_{1}, i_{2}\right) \in \Lambda .
$$

Here, $g_{i_{1}, i_{2}}$ represents the intensity of the image $\mathbf{g}$ at the pixel $\left(i_{1}, i_{2}\right)$. We will use the same convention, the use of boldface alphabet for the image and the normal alphabet with the pixel subscript for the intensity, throughout this paper. Notations $\ell^{2}(\Omega)$ and $\ell^{2}(\Lambda)$ are used to denote image spaces defined on $\Omega$ and $\Lambda$, respectively. Here, inner products of $\ell^{2}(\Omega)$ and $\ell^{2}(\Lambda)$ are the usual dot product of two images (sums of pixel-by-pixel multiplications).

In (1), we assume that the blurring by $\mathcal{T}$ represents a truncated convolution with a known PSF $\mathbf{k}=\left(k_{i_{1}, i_{2}}\right)$ : For any image $\mathbf{p} \in \ell^{2}(\Omega)$,

$$
(\mathcal{T} \mathbf{p})_{i_{1}, i_{2}}=\sum_{\left(j_{1}, j_{2}\right) \in\left(i_{1}, i_{2}\right)-\mathcal{S}_{\mathbf{k}}} k_{i_{1}-j_{1}, i_{2}-j_{2}} p_{j_{1}, j_{2}} .
$$

Here $\mathcal{S}_{\mathbf{k}}$, the support of $\mathbf{k}$, is $\left\{\left(j_{1}, j_{2}\right) \mid k_{j_{1}, j_{2}}>0\right\}$. We also assume that the PSF $\mathbf{k}$ is nonnegative, its components have sum 1 , and the point $(0,0) \in \mathcal{S}_{\mathbf{k}} ; \mathcal{T} \mathbf{p}$ is defined on $\Lambda$, where $\left(i_{1}, i_{2}\right) \in \Lambda$ if and only if $\left(i_{1}, i_{2}\right)-\mathcal{S}_{\mathbf{k}} \subset \Omega$. Thus, $\Lambda \subset \Omega$.

\subsection{Computation of $\mathcal{T}$ and $\mathcal{T}^{*}$}

Let $\mathcal{T}^{*}$ be the adjoint operator of $\mathcal{T}$ in (2), i.e., $\mathcal{T}^{*}$ is defined by the relation

$$
\mathbf{q} \cdot(\mathcal{T} \mathbf{p})=\left(\mathcal{T}^{*} \mathbf{q}\right) \odot \mathbf{p}
$$

for $\mathbf{q} \in \ell^{2}(\Lambda)$ and $\mathbf{p} \in \ell^{2}(\Omega)$, and hence $\mathcal{T}^{*}: \ell^{2}(\Lambda) \rightarrow$ $\ell^{2}(\Omega)$. Here, notations $\cdot$ and $\odot$ were used to denote dot products of images defined on $\Lambda$ and $\Omega$, respectively.

The computation of $\mathcal{T} \mathbf{p}$ can be carried out by the pixelwise definition (2) or by using the fast Fourier transform (FFT) with a zero padding. In the case when the pixel-wise definition is used for $\mathcal{T} \mathbf{p}$, then

$$
\text { OC(pixel-wise computation of } \mathcal{T} \mathbf{p})=|\Lambda| \cdot\left|\mathcal{S}_{\mathbf{k}}\right|,
$$

since $\left|\mathcal{S}_{\mathbf{k}}\right|$ operations (one operation $=$ one multiplication + one addition) are required for the computation of $(\mathcal{T} \mathbf{p})_{i_{1}, i_{2}}$ for each $\left(i_{1}, i_{2}\right) \in \Lambda$. Here, $\mathbf{O C}$ means operation counts. On the other hand, with the assumption $\left|\mathcal{S}_{\mathbf{k}}\right|<$ $|\Omega|$,

$$
\text { OC }(\text { FFT computation of } \mathcal{T} \mathbf{p}) \approx|\Omega| \log _{2}|\Omega| \text {. }
$$

It is not difficult to show that $\mathcal{T}^{*}$ can be computed by

$$
\left(\mathcal{T}^{*} \mathbf{q}\right)_{j_{1}, j_{2}}=\sum_{\left(i_{1}, i_{2}\right) \in\left(\left(j_{1}, j_{2}\right)+\mathcal{S}_{\mathbf{k}}\right) \cap \Lambda} k_{i_{1}-j_{1}, i_{2}-j_{2}} q_{i_{1}, i_{2}},
$$

for $\mathbf{q} \in \ell^{2}(\Lambda)$.

Thus,

OC(pixel-wise computation of $\left.\mathcal{T}^{*} \mathbf{q}\right)=|\Lambda| \cdot\left|\mathcal{S}_{\mathbf{k}}\right| \cdot$

It is also possible that the computation of $\mathcal{T}^{*} \mathbf{q}$ can be carried out by using FFT with a zero padding. In that case, with the assumption $\left|\mathcal{S}_{\mathbf{k}}\right|<|\Omega|$,

$$
\text { OC(FFT computation of } \left.\mathcal{T}^{*} \mathbf{q}\right) \approx|\Omega| \log _{2}|\Omega| \text {. }
$$

\subsection{Richardson-Lucy iteration}

For the image deblurring problem (1), one iteration of RL takes

$$
\mathbf{f}^{n+1}=\mathbf{f}^{n} \cdot * \frac{\mathcal{T}^{*} \mathbf{s}^{n}}{\mathcal{T}^{*} \mathbf{I}_{\Lambda}}, \quad \mathbf{s}^{n}=\frac{\mathbf{g}}{\mathcal{T} \mathbf{f}^{n}} .
$$

Here, the notation $\mathbf{I}_{A}$ means the all-one image on the pixel subset $A$ and .* is the pixel-by-pixel multiplication, and the division between two images, $\frac{\mathbf{g}}{\mathcal{T} \mathbf{f}^{n}}$, is the image resulted from the pixel-by-pixel division of $\mathbf{g}$ and $\mathcal{T} \mathbf{f}^{n}$.

For future use, one-step iteration of RL (8) will be denoted by

$$
\mathbf{f}^{n+1}=\mathbf{R} \mathbf{L}\left(\mathbf{g}, \mathbf{f}^{n}, \mathbf{k}, \Lambda\right) .
$$

Here, the PSF $\mathbf{k}$ was used instead of $\mathcal{T}$ and $\mathcal{T}^{*}$.

Performing RL as described in (8) often results in a very slow convergence. For the acceleration of RL, several methods have been suggested [7, 19-21]. Among them, the technique in [7] has been noted for its success. For example, the function 'deconvlucy', the RL implementation 
of the software MATLAB ${ }^{ø}$ (The MathWorks, Natick, MA, USA), uses the technique described in Section 2.3 and 2.4 in [7]. Interlaced BI-RL, the proposed method for the acceleration of RL in this paper, will be compared with the technique in [7] in Section 4.

\subsection{Boundary artifacts}

Boundary artifacts are one of key obstacles in the development of BI-RL; block boundaries introduced by the block partition may cause artifacts in combining deblurred block images. It is also true that boundary artifacts are one of key obstacles in many image deblurring problems [22].

To reduce boundary artifacts in image deblurring, many methods have been proposed. One group of methods imposes certain conditions on pixels in $\Omega-\Lambda$. Examples include periodic, reflective, and anti-reflective boundary conditions [23-27].

Other group of methods [17, 28, 29] does not impose any conditions on pixels in $\Omega-\Lambda$, and let the iteration itself determine results in $\Omega-\Lambda$. In [29], this approach is called the free boundary condition method.

Before we close this section, it is worth to mention some research works related to fast direct deblurring methods. It is well known that if the imposed boundary condition is one of periodic, reflective, or anti-reflective boundary conditions, then the image deblurring with symmetric PSFs (for a periodic boundary condition, the symmetry of PSF can be omitted) can be directly computable by using FFT for periodic boundary condition, discrete cosine transform (DCT) for reflective boundary condition, and discrete sine transform (DST) for anti-reflective boundary condition $[1,23,25]$.

These fast transform-based direct deblurring methods, however, often present severe boundary artifacts. To reduce boundary artifacts, one can smooth the boundaries of the observed image to decay to 0 (to make the imposed boundary condition to be more feasible) before those direct deblurring methods are applied to. This approach can reduce boundary artifacts in some degree, but, at the same time, makes it more difficult to recover near boundary image pixels.

The performance of direct deblurring methods depends heavily on the feasibility of imposed boundary conditions. The difficulty of imposing correct boundary conditions is the main reason why iterative deblurring approaches with free boundary conditions have been considered, despite the fact that fast direct deblurring methods are available $[17,28,29]$.

Considering these facts, we suggest the free boundary condition-based RL approach for the image deblurring problem (1). To reduce computational burden and accelerate the slow convergence of $\mathrm{RL}$, we will propose block-iterative methods in the next section.

\section{Proposed method}

\subsection{Block partition}

BI-RL decomposes the original deblurring problem $\mathbf{g}=$ $\mathcal{T}$ f (here the noise term is ignored for the simplicity of the presentation) into several (say $t$ ) block deblurring problems

$$
\mathbf{g}^{[i]}=\mathcal{T}_{i} \mathbf{f}^{[i]}, \quad i=1, \ldots, t,
$$

by partitioning $\Lambda$ into $\Lambda_{i}$, where $\mathbf{g}^{[i]}=\left.\mathbf{g}\right|_{\Lambda_{i}}$, the restriction of $\mathbf{g}$ on $\Lambda_{i}$, and $\mathcal{T}_{i}=\left.\mathcal{T}\right|_{\Omega_{i}}$, the restriction of $\mathcal{T}$ on some subset $\Omega_{i} \subset \Omega$. Since only pixels that contribute to the observation on $\Lambda_{i}$ can be recovered in (9), the subset $\Omega_{i}$ can be defined by

$$
\Omega_{i}=\left\{\left(j_{1}, j_{2}\right) \in \Omega \mid\left(\mathcal{T}^{*} \mathbf{I}_{\Lambda_{i}}\right)_{j_{1}, j_{2}}>0\right\}
$$

for $i=1,2, \ldots, t$. Notice that pixels only in $\Omega_{i}$ can contribute the observation $\mathbf{g}^{[i]}$ (defined on $\Lambda_{i}$ ) and $\Lambda_{i} \subset \Omega_{i}$ for $i=1,2, \ldots, t$.

Throughout this paper, $\Lambda_{i}$ are assumed to be mutually disjoint, unless stated otherwise.

In any cases, $\Lambda_{i}$ are selected to satisfy

$$
\Lambda=\cup_{i=1}^{t} \Lambda_{i} \text { and } \Omega=\cup_{i=1}^{t} \Omega_{i} \text {. }
$$

\subsection{Separated BI-RL}

Separated BI-RL: Given $N$,

$$
\begin{array}{ll}
\mathbf{S 1} & \text { for } i=1,2, \ldots, t \\
\mathbf{S 2} & \mathbf{f}^{[i], 0}=\mathbf{I}_{\Omega_{i}} \\
\mathbf{S 3} & \text { for } m=0,1, \ldots, N-1 \\
\mathbf{S 4} & \mathbf{f}^{[i], m+1}=\mathbf{R} \mathbf{L}\left(\mathbf{g}^{[i]}, \mathbf{f}^{[i], m}, \quad \mathbf{k}, \Lambda_{i}\right) \\
\mathbf{S 5} & \text { end } \\
\mathbf{S 6} & \text { end } \\
\mathbf{S 7} & \hat{\mathbf{f}}=\mathbf{r}^{[1]} \cdot * \mathbf{f}^{[1], N}+\ldots+\mathbf{r}^{[t]} * * \quad \mathbf{f}^{[t], N}
\end{array}
$$

In the step $\mathbf{S} 7$, weights $\mathbf{r}^{[i]}$ are defined by

$$
\mathbf{r}^{[i]}=\frac{\mathcal{T}^{*} \mathbf{I}_{\Lambda_{i}}}{\sum_{i=1}^{t} \mathcal{T}^{*} \mathbf{I}_{\Lambda_{i}}} .
$$

The weights $\mathbf{r}^{[i]}$ in (11) are motivated by the following interpretation. Recall that $\mathcal{T}$ represents the truncated convolution by the PSF $\mathbf{k}$ that is nonnegative and has 1 as its sum of all components. Thus, with the assumption that mutually disjoint blocks $\Lambda_{i}, i=1,2, \ldots, t$ are selected to form $\Lambda=\cup_{i=1}^{t} \Lambda_{i},\left(\mathcal{T}^{*} \mathbf{I}_{\Lambda_{i}}\right)_{j_{1}, j_{2}}$ can be interpreted as the probability with which the pixel $\left(j_{1}, j_{2}\right) \in \Omega$ contributes the observation on $\Lambda_{i}$. This argument shows that weights $\mathbf{r}^{[i]}$ make the intensity at the pixel $\left(j_{1}, j_{2}\right)$ to depend on $\mathbf{f}^{[i], N}$ (the deblurred image from $\mathbf{g}^{[i]}$ defined on $\Lambda_{i}$ ) proportionally to the probability of the contribution of the pixel $\left(j_{1}, j_{2}\right)$ to $\mathbf{g}^{[i]}$ (the observation on $\Lambda_{i}$ ).

For certain deblurring problems, it is desirable to use overlapped blocks $\Lambda_{i}$. In such case, separated BI-RL separately deblurs $\mathbf{g}^{[i]}$ on overlapped blocks $\Lambda_{i}$ to produce 
a deblurred block image $\mathbf{f}^{[i], N}$ for each $i$, and, after cropping out some of overlapped pixels of $\mathbf{f}^{[i], N}, i=1, \ldots, t$, combines them to the final output image.

\subsection{Interlaced BI-RL}

Interlaced BI-RL: Given $N$,

$$
\begin{aligned}
& \text { I1 } \mathbf{f}^{0}=\mathbf{R L}\left(\mathbf{g}, \mathbf{I}_{\Omega}, \mathbf{k}, \Lambda\right) \\
& \text { I2 for } m=0,1,2, \ldots, N-1 \\
& \text { I3 for } i=1,2, \ldots, t \\
& \left.\mathbf{I 4} \quad \mathbf{f}^{m+i / t}\right|_{\Omega_{i}}=\mathbf{R} \mathbf{L}\left(\mathbf{g}^{[i]},\left.\mathbf{f}^{m+(i-1) / t}\right|_{\Omega_{i}}, \mathbf{k}, \Lambda_{i}\right) \text {; } \\
& \text { I5 end } \\
& \text { I6 end } \\
& \text { I7 } \hat{\mathbf{f}}=\mathbf{f}^{N}
\end{aligned}
$$

To explain a key point of interlaced BI-RL, let us compare interlaced BI-RL with RL. Notice that RL can be obtained from interlaced BI-RL by replacing the inner for loop (steps I3, I4, and I5) with $\mathbf{f}^{m+1}=\mathbf{R} \mathbf{L}\left(\mathbf{g}, \mathbf{f}^{m}, \mathbf{k}, \Lambda\right)$. Suppose that selected blocks $\Lambda_{i}, i=1,2, \ldots, t$, satisfy

$$
\Lambda \subset \cap_{i=1}^{t} \Omega_{i} \approx \Omega
$$

where $\Omega_{i}$ is computed by (10) from $\Lambda_{i}$. In this case, one iteration of interlaced BI-RL (from steps I3 to I5) updates most pixel values on $\Omega$, including all pixel values on $\Lambda$, $t$ times, while RL updates pixel values on $\Omega$ just once. Notice that, in the algorithmic point of view, the described benefit of interlaced BI-RL is identical to that of OSEM [6] in emission tomography.

$\mathrm{RL}$ often uses the all-one image $\mathbf{I}_{\Omega}$ as the initial guess $\mathbf{f}^{0}$. Interlaced BI-RL, however, uses $\mathbf{f}^{0}=\mathbf{R} \mathbf{L}\left(\mathbf{g}, \mathbf{I}_{\Omega}, \mathbf{k}, \Lambda\right)$ as the initial guess (see the step I1). This suggestion is made to update pixel values of $\mathbf{f}^{0}$ on $\Omega_{i}$, without causing many discontinuities.

Figure 1 illustrates the procedure of interlaced BI-RL. In Fig. 1, the observed image $\mathbf{g}$ is decomposed into four block images $\mathbf{g}^{[i]}, i=1,2,3,4$, defined on $2 \times 2$ rectangularlydown-sampled blocks $\Lambda_{i}, i=1,2,3,4$. The initial guess $\mathbf{f}^{0}$ is computed by $\mathbf{R L}\left(\mathbf{g}, \mathbf{I}_{\Omega}, \mathbf{k}, \Lambda\right)$. In $m=0$ and $i=1$, the step $\mathbf{I 4}$ updates pixel values of $\mathbf{f}^{0}$ on $\Omega_{1}$ by $\mathbf{R} \mathbf{L}\left(\mathbf{g}^{[1]},\left.\mathbf{f}^{0}\right|_{\Omega_{1}}\right.$, $\left.\mathbf{k}, \Lambda_{1}\right)(\mathbf{I 4})$. The resulting image is denoted by $\mathbf{f}^{1 / 4}$. This procedure makes pixel values of $\mathbf{f}^{1 / 4}$ on $\Omega_{1}$ to be already quite close to the true image $\mathbf{f}$ at pixels on $\Omega_{1}$, but it does not change pixel values on $\Omega-\Omega_{1}$, i.e., $\mathbf{f}^{1 / 4}=\mathbf{f}^{0}$ on $\Omega-\Omega_{1}$. Thus, the step I4 would cause many discontinuities if the all-one image $\mathbf{I}_{\Omega}$ were selected for the initial guess $\mathbf{f}^{0}$.

In the next sub-iteration ( $m=0$ and $i=2)$, pixel values on $\Omega_{2}$ are updated by $\mathbf{R L}\left(\mathbf{g}^{[2]},\left.\mathbf{f}^{1 / 4}\right|_{\Omega_{2}}, \mathbf{k}, \Lambda_{2}\right)$. Once pixel values are updated on remaining blocks, $\Omega_{3}$ and $\Omega_{4}$, the resulting image is $\mathbf{f}^{1}$ as shown in Fig. 1.

\subsection{Algorithmic limitations of proposed methods}

In the algorithmic point of view, separated BI-RL can be used for any PSFs. Roughly speaking, the size of the PSF determines the minimum size of blocks that can be used for separated BI-RL, and the number of blocks determines the maximum gain in separated BI-RL by parallel computations.

The usefulness of interlaced BI-RL, however, is limited to PSFs with small number of non-zero elements. To explain this, let us recall that interlaced BI-RL uses pointwise computations for convolutions, (2) and (5). Thus, one iteration of interlaced BI-RL with $t$ down-sampled blocks requires $2\left|\mathcal{S}_{\mathbf{k}}\right| \cdot|\Lambda|+2 t|\Omega|$ operation counts, where $\left|\mathcal{S}_{\mathbf{k}}\right|$ is the number of non-zero elements in the PSF $\mathbf{k}$. On the other hand, one iteration of FFT-based RL requires $2 C|\Omega| \log _{2}|\Omega|+|\Omega|$ operation counts, where $C$ is a constant which depends on the way of implementing FFT algorithm. Therefore, in order for interlaced BI-RL with $t$ down-sampled blocks to be useful as compared with FFT-based RL, the PSF k must satisfy

$$
\left(2\left|\mathcal{S}_{\mathbf{k}}\right| \cdot|\Lambda|+2 t|\Omega|\right) / t \leq 2 \mathrm{C}|\Omega| \log _{2}|\Omega|+|\Omega|,
$$

where the denominator $t$ in the left-hand side is used by considering that interlaced BI-RL with well chosen $t$ down-sampled blocks accelerates iterations $t$ times. The condition (13) does not hold for PSFs with large numbers of non-zero elements.

In our simulation, we used PSFs $\mathbf{k}$ with $\left|\mathcal{S}_{\mathbf{k}}\right| \leq 441$ and $480 \times 480$ sized images. For such PSFs and images, the FFT-based RL implementation optimized for $512 \times 512$ sized images was slower than the pixel-wise computationbased RL implementation. The condition $\left|\mathcal{S}_{\mathbf{k}}\right| \leq 441$ includes PSFs that are used in many important image deblurring applications. For instance, any PSFs that have not more than 441 non-zero elements (e.g., a PSF of the form of the $441 \times 441$ diagonal matrix) satisfy this condition. Thus, interlaced BI-RL is computationally more efficient than FFT-based RL in those image deblurring applications.

\subsection{Free boundary condition}

Proposed methods use the free boundary condition to suppress block artifacts. This suggestion is based on the observation that the free boundary condition successfully suppresses boundary artifacts for arbitrarily shaped images; note that periodic, reflective, and anti-reflective boundary conditions can be applied to rectangularshaped images only. For details, see [23-26].

The use of the free boundary condition appears in the step $\mathbf{S 4}$ and the step $\mathbf{I 4}$ by imposing no restriction on pixels on $\Omega_{i}-\Lambda_{i}$. The step $\mathbf{I} 1$ of interlaced BI-RL also uses the free boundary condition, again, by imposing no restriction on pixels on $\Omega-\Lambda$. 


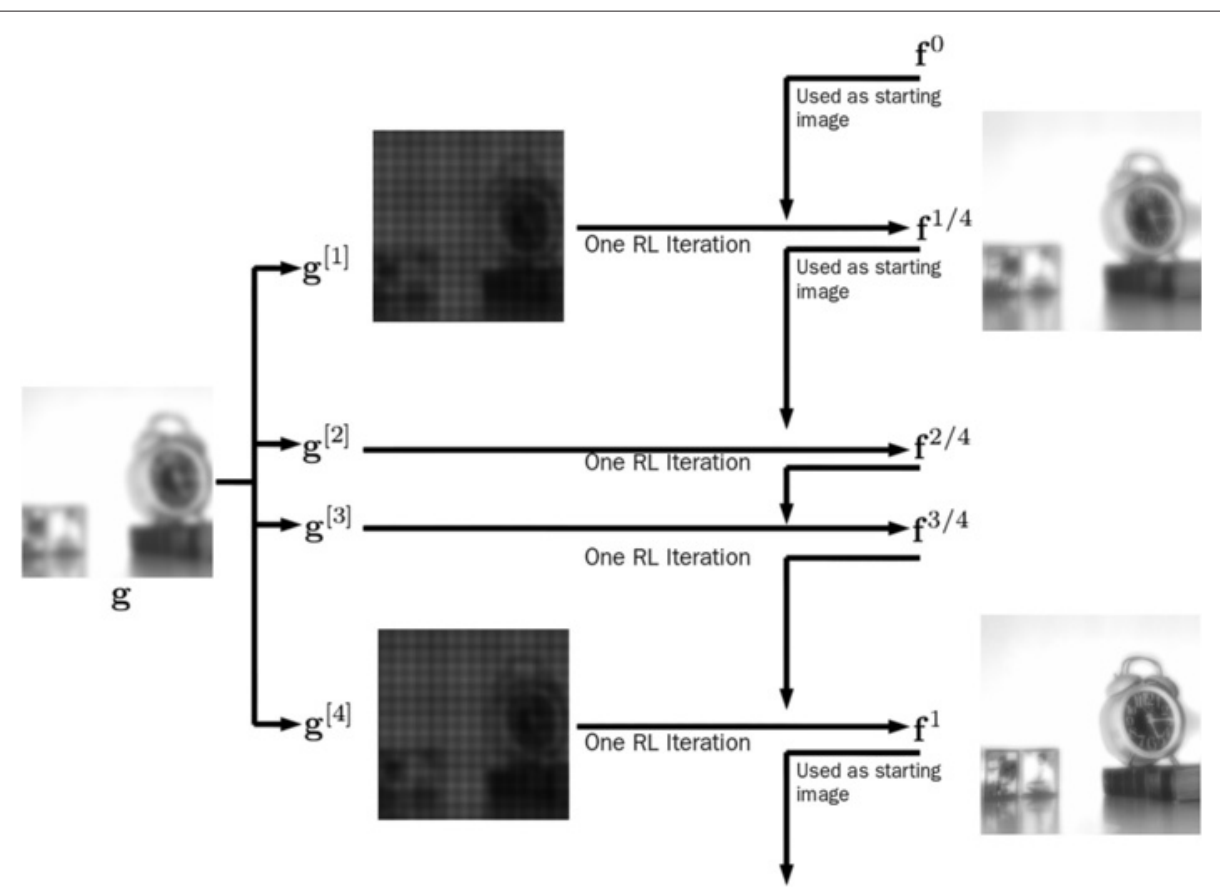

Fig. 1 Interlaced BI-RL with $2 \times 2$ rectangularly-down-sampled blocks. The observed image $\mathbf{g}$ of size $200 \times 200$, which is blurred by a Gaussian PSF $\mathbf{k}$ of size $41 \times 41$, is decomposed to $2 \times 2$ rectangularly-down-sampled blocks. The starting image $\mathbf{f}^{0}$ is computed from $\mathbf{g}$ by RL. The first sub-iteration of interlaced BI-RL updates pixel values on $\Omega_{1}$ by using $\mathbf{g}^{[1]}$ as the observed data and $\mathbf{f}^{0}$ as the starting image. The resulting image of this process is $\mathbf{f}^{1 / 4}$. The second sub-iteration of interlaced BI-RL updates pixel values on $\Omega_{2}$ by using $\mathbf{g}^{[2]}$ as the observed data and $\mathbf{f}^{1 / 4}$ as the starting image. The resulting image of this process is $\mathbf{f}^{2 / 4}$. Similarly, the third and fourth sub-iterations of interlaced BI-RL update pixel values on $\Omega_{3}$ and $\Omega_{4}$. This completes one iteration of interlaced BI-RL with four blocks

\subsection{Examples of blocks}

Examples of blocks in this section are selected by using following two suggestions:

- For separated BI-RL, select blocks $\Lambda_{i}, i=1,2, \ldots, t$, that make $\Omega_{j} \cap \Omega_{n}$ as small as possible for all pairs $(j, n), 1 \leq j<n \leq t$.

- For interlaced BI-RL, select the block $\Lambda_{i}$ that makes $\Omega_{i} \approx \Omega$ and $\mathcal{T}^{*} \mathbf{I}_{\Lambda_{i}}$ to be uniform as much as possible for each $i$.

Simulation results in Section 4 will show why these two suggestions are important.

The work in this paper will be restricted to the test of following blocks (illustrated in Fig. 2) in deblurring problems modeled by the Gaussian PSF $\mathbf{k}_{G}$ (Fig. 3a) and the diagonal PSF $\mathbf{k}_{D}$ (Fig. 3b).

\subsubsection{Rectangular blocks}

Figure 2a shows $4 \times 4$ rectangular blocks of the observed image (Fig. 5a). Separated BI-RL with $2 \times 2,4 \times 4,8 \times 8$, and $16 \times 16$ rectangular blocks will be tested in the Gaussian deblurring.

Overlapped rectangular blocks can be formed by adding several pixel rows and columns to boundaries of disjoint rectangular blocks. This type of overlapped blocks will be also tested in separated BI-RL.

\subsubsection{Diagonal blocks}

Figure 2b shows 8 diagonal blocks of the observed image (Fig. 5b). Separated BI-RL with 2, 4, 8, and 16 diagonal blocks will be tested in the diagonal deblurring.

\subsubsection{Rectangularly-down-sampled blocks}

Figure 2(c) shows $4 \times 4$ rectangularly-down-sampled blocks of the observed image (Fig. 5a). To be specific, if the pixel index at the left and upper corner of the observed image is $(0,0)$, then $4 \times 4$ down-sampled blocks are defined by

$$
\Lambda_{4 y+x+1}=\left\{\left(i_{1}, i_{2}\right) \in \Lambda \mid i_{1} \in 4 \mathbb{Z}+y, i_{2} \in 4 \mathbb{Z}+x\right\},
$$

for $x, y=0,1,2,3$, where $4 \mathbb{Z}$ is the integer subset formed by multiples of 4 . With the same argument, other rectangularly-down-sampled blocks can be defined. Interlaced BI-RL with $2 \times 2,4 \times 4,6 \times 6$, and $8 \times 8$ rectangularly-down-sampled blocks will be tested in the Gaussian deblurring.

\subsubsection{Diagonally-down-sampled blocks}

Figure 2d shows 8 diagonally-down-sampled blocks of the observed image (Fig. 5b).

To be specific,

$$
\Lambda_{n+1}=\left\{\left(i_{1}, i_{2}\right) \in \Lambda \mid i_{1}+i_{2} \in 8 \mathbb{Z}+n\right\},
$$



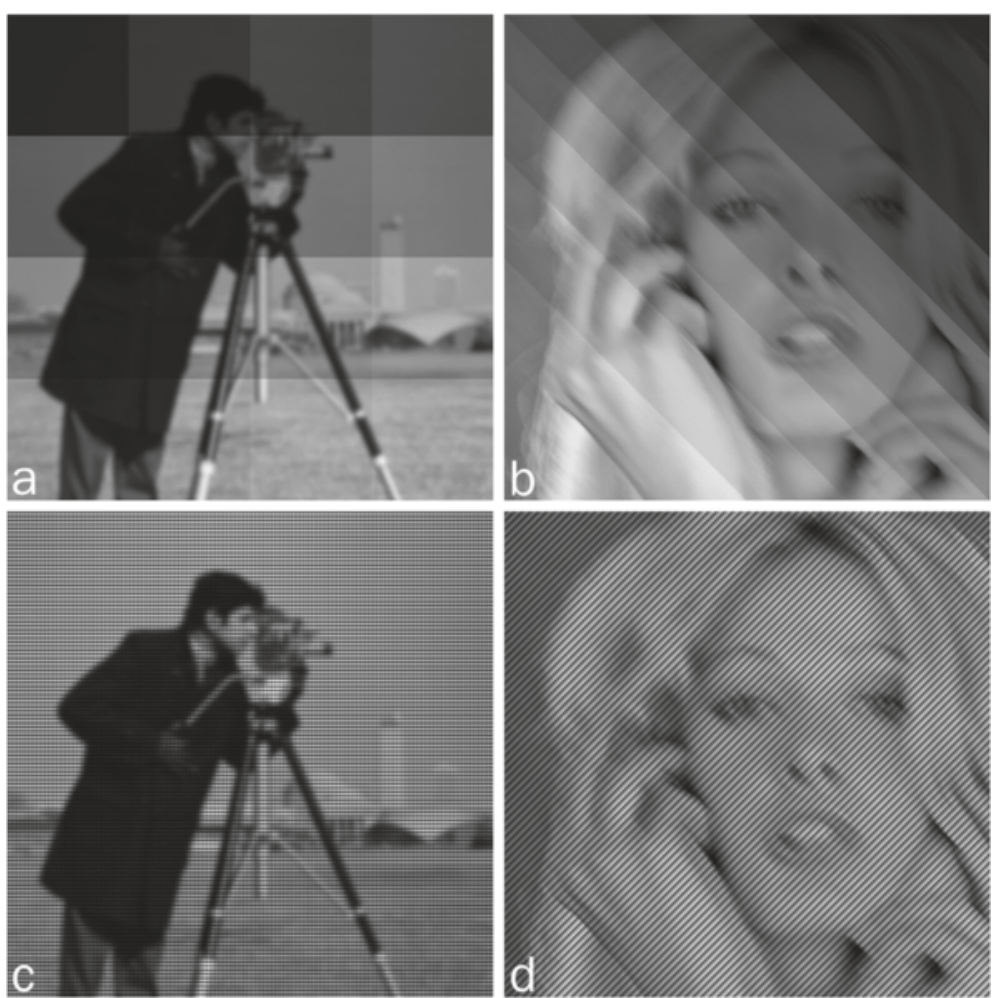

Fig. 2 Examples of block partitions. Different intensity scales were used to visualize block partitions. a $4 \times 4$ rectangular blocks, b 8 diagonal blocks, c $4 \times 4$ rectangularly-down-sampled blocks, and $\mathbf{d} 8$ diagonally-down-sampled blocks

for $n=0,1, \ldots, 7$, form 8 diagonally-down-sampled blocks.

Interlaced BI-RL with 2, 4, 6, and 8 diagonally-downsampled blocks will be tested in the diagonal deblurring.

\section{Simulation results and discussion}

We conducted simulation studies to test the performance of proposed methods in Gaussian and diagonal deblurrings. In simulation studies, 'cameraman' (Fig. 4a) and 'girl' (Fig. 4b), of size $500 \times 500$, were used as true images. PSFs
$\mathbf{k}_{G}$ and $\mathbf{k}_{D}$ in Fig. 3 were applied to 'cameraman' and 'girl', respectively, to produce blurred images of size $480 \times 480$ (both PSFs are of size $21 \times 21$ ).

Blurred images were further corrupted by the Poissonian noise model in (1). Total sums of intensities of noisy blurred images (Fig. 5) were 2.6 and 3.1 billions for 'cameraman' and 'girl', respectively.

As mentioned in Section 3, interlaced BI-RL is useful only when pixel-wise computations (2) and (5), instead of FFT-based ones, are used for $\mathcal{T}$ and $\mathcal{T}^{*}$. In simulation
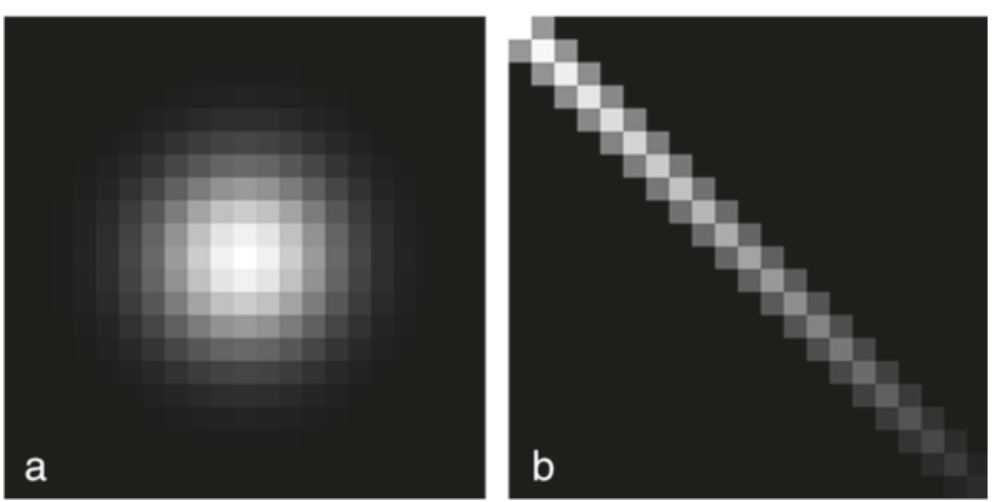

Fig. 3 PSFs. Both PSFs are of size $21 \times 21$. a Gaussian PSF $\mathbf{k}_{G}$ and $\mathbf{b}$ diagonal PSF $\mathbf{k}_{D}$ 


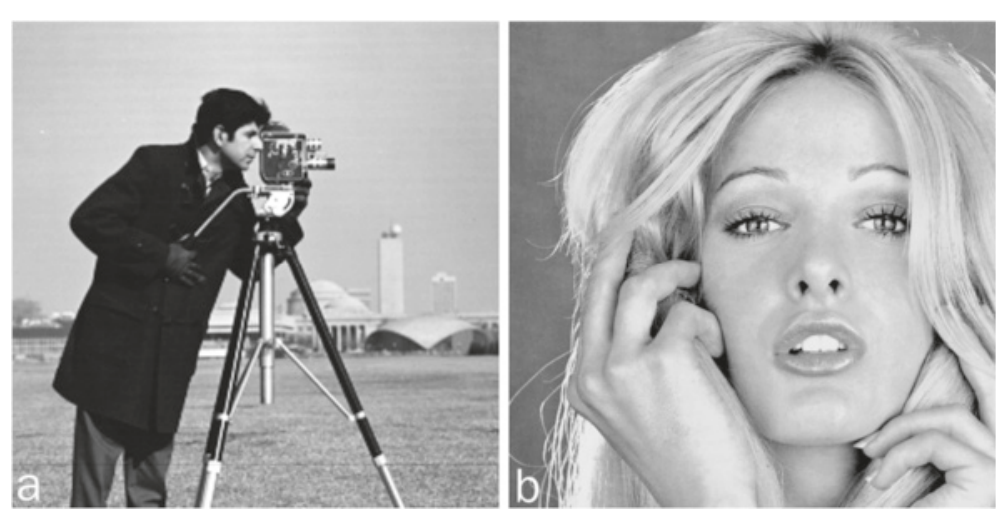

Fig. 4 Test images. Both images are of size $500 \times 500$. a 'Cameraman' and b 'girl'

studies, we used a personal computer equipped with 2.0 GHz Intel Core 2 Duo CPU and 8 GB RAM. In this computing environment, one RL iteration with FFT-based computations took $5.04 \mathrm{~s}$ both for Gaussian and diagonal deblurrings. On the other hand, one round of interlaced BI-RL (the for-loop in steps I3, I4, and I5) with pixel-wise computations took $4.84 \mathrm{~s}$ for $4 \times 4$ rectangularly-downsampled blocks for Gaussian deblurring $\left(\left|\mathcal{S}_{\mathbf{k}_{G}}\right|=441\right)$ and $0.88 \mathrm{~s}$ for 8 diagonally-down-sampled blocks for the diagonal deblurring $\left(\left|\mathcal{S}_{\mathbf{k}_{D}}\right|=61\right)$. This result implies that interlaced BI-RL is, at least computational point of view, useful for both deblurring problems in our simulation. Considering these facts, we used pixel-wise computations (2) and (5) in our simulation.

Based on the experience that it is not easy to choose an un-biased stopping rule, we selected the image that had the smallest relative square error (RSE) within 1000 iterations as the deblurred image of the tested method. Here, the RSE is defined by

$$
\operatorname{RSE}=\frac{\sum_{\left(i_{1}, i_{2}\right) \in \Lambda}\left|\hat{f}_{i_{1}, i_{2}}-f_{i_{1}, i_{2}}\right|^{2}}{\sum_{\left(i_{1}, i_{2}\right) \in \Lambda}\left|f_{i_{1}, i_{2}}\right|^{2}},
$$

where $\hat{f}_{i_{1}, i_{2}}$ and $f_{i_{1}, i_{2}}$ are intensities of the deblurred image and the true image, respectively.

\subsection{Standard RL}

Figure $6 \mathrm{a}, \mathrm{b}$ shows deblurred images by RL from Figs. 5a and $5 \mathrm{~b}$, respectively. Figure $6 \mathrm{a}$ was obtained by 420 iterations with RSE $=0.52 \%$ and Fig. $6 \mathrm{~b}$ by 102 iterations with $\mathrm{RSE}=0.65 \%$. All simulation results by BI-RL will be compared with these images.

\subsection{Separated BI-RL}

\subsubsection{Rectangular blocks for Gaussian deblurring}

Table 1 shows results of separated BI-RL with rectangular blocks in the Gaussian deblurring. For instance, computation times used for the block partition ('BP' column), the single block iteration ('SB' column), and the combining of deblurred block images ('CB' column) were listed in Table 1. The smallest RSEs and their corresponding iteration numbers ('IN' column) were also listed in Table 1.

As 'the number of blocks' (hereafter abbreviated by NB) increased, computation times for the block partition (BP) and the combining of block images (CB) increased.

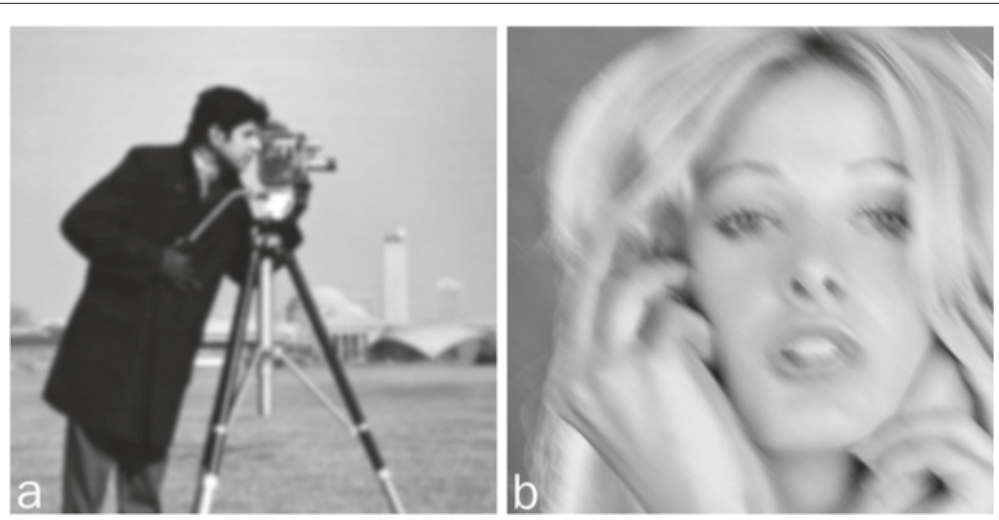

Fig. 5 Noisy, blurred images. Both images are of size $480 \times 480$. These images were obtained by applying the Gaussian PSF $\mathbf{k}_{G}$ (a) and the diagonal PSF $\mathbf{k}_{D}(\mathbf{b})$ to true images in Fig. 4 and adding Poisson noises to have total sums of all pixel values of 'cameraman' (a) and 'girl' (b) to be 2.6 and 3.1 billions, respectively 


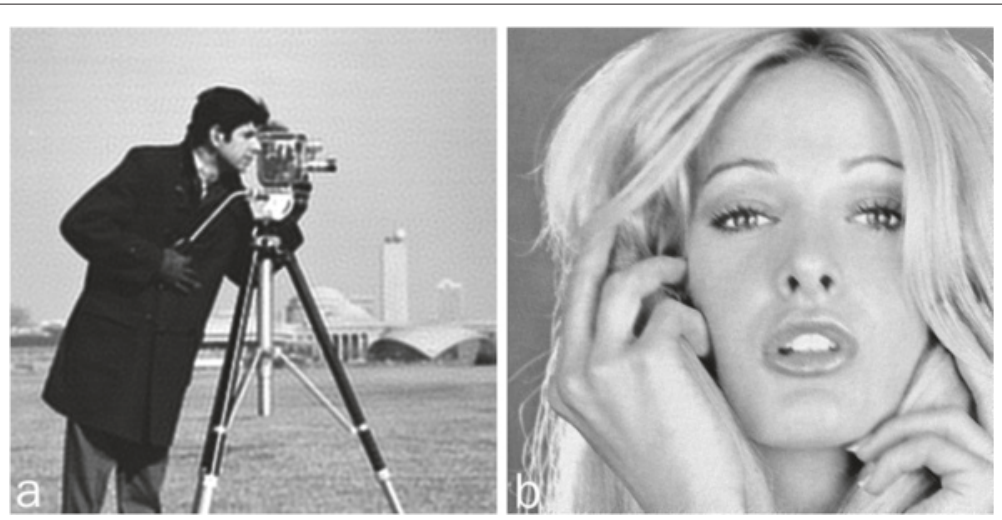

Fig. 6 Deblurred images by RL. These images were obtained at the 420th iteration with RSE $=0.52 \%$ for $(\mathbf{a})$ and 102 th iteration with RSE $=0.65 \%$ for $(\mathbf{b})$

Increments in computation times were, however, very small. The time for the single block iteration, however, linearly decreased as NB increased. There were also some slight increments in RSE and IN as NB increased.

Figure 7 shows deblurred images by separated BI-RL with $4 \times 4($ a) and $16 \times 16$ (b) rectangular blocks. Figure $7 \mathrm{a}$ was obtained at the 432th iteration with RSE $=0.53 \%$ and Fig. $7 \mathrm{~b}$ at the 513th iteration with RSE $=0.55 \%$. No noticeable block artifacts are shown in Fig. 7a, b. As differences in RSE results in Table 1 might indicate, Fig. 7b looks slightly smoother than Fig. 7a, while Fig. 7a looks slightly smoother than Fig. 6a. As shown in Fig. 7a, however, the degradation in Fig. 7a in the comparison with Fig. 6a is not big enough to give up the efficiency of separated BI-RL in parallel computations. See results in the 'PC' column in Table 1.

Results in Table 1 and Fig. 7 show that separated BI-RL with, at least up to $4 \times 4$, rectangular blocks achieves the desired goal (combining deblurred block images to final images without block artifacts, while maintaining deblurring quality and approximation rate) in the Gaussian deblurring.

Table 1 Computation times for separated BI-RL with rectangular blocks for the Gaussian deblurring and their smallest RSE results

\begin{tabular}{ccccccc}
\hline NB & BP & SB & CB & RSE $(\%)$ & IN & PC \\
\hline $1 \times 1$ & n.a. & 4.400 & n.a. & 0.52 & 420 & 1,848 \\
$2 \times 2$ & 10.1 & 1.098 & 0.0080 & 0.53 & 441 & 494 \\
$4 \times 4$ & 10.3 & 0.275 & 0.0093 & 0.53 & 432 & 129 \\
$8 \times 8$ & 10.6 & 0.068 & 0.0126 & 0.54 & 499 & 44 \\
$16 \times 16$ & 11.6 & 0.017 & 0.0208 & 0.55 & 513 & 20
\end{tabular}

Here, the following abbreviations were used: NB number of blocks, n.a. not applied, $B P$ seconds spent for block partitioning, SB seconds spent for the single block iteration, $C B$ seconds spent for the combining of deblurred block images, IN the iteration number that attained the smallest RSE, and $P C$ seconds spent for the parallel computation in case when NB processing units are used. The row starting with ' $1 \times 1$ ' in NB column represents results by RL

\subsubsection{Diagonal blocks for diagonal deblurring}

Table 2 shows the same data as Table 1 for diagonal blocks for diagonal deblurring. As in Table 1, the computation time for the single block iteration linearly decreases as NB increases, while there are some negligible increments in computation times for the block partition and the combining of deblurred block images. Unlike in Table 1, however, RSE and IN results are virtually unchanged as NB increases.

Figure 8 shows deblurred images by separated BI-RL with 8 (a) and 16 (b) diagonal blocks. Figure 8a was obtained at the 103th iteration with RSE $=0.65 \%$ and Fig. $8 \mathrm{~b}$ at the 104th iteration with RSE $=0.65 \%$. Again, no noticeable boundary artifacts are shown in Fig. 8. As RSE results in Table 2 might indicate, visual differences in Figs. $6 \mathrm{~b}$ and $8 \mathrm{a}, \mathrm{b}$ are hardly noticeable.

Results in Table 2 and Fig. 8 show that separated BI$\mathrm{RL}$ with, at least up to 16, diagonal blocks achieves the desired goal (combining deblurred block images to final images without block artifacts, while maintaining deblurring quality and approximation rate) in the diagonal deblurring.

\subsection{Interlaced BI-RL}

\subsubsection{Rectangularly-down-sampled blocks for Gaussian deblurring}

Table 3 shows results of interlaced BI-RL with rectangularly-down-sampled blocks in the Gaussian deblurring. The computation time in the 'BI' column slightly increases as NB increases; one RL iteration took $4.40 \mathrm{~s}$, while the one round of interlaced BI-RL iteration with $4 \times 4$ rectangularly-down-sampled blocks took $4.84 \mathrm{~s}$ (see Table 3). The increment from 4.40 to $4.80 \mathrm{~s}$ was caused by updating pixel values on $\Omega_{i}$ for all $i=1,2, \ldots, 16$ (see step I4) in interlaced BI-RL.

RSE results in Table 3 show that as NB increases, interlaced BI-RL reaches its smallest RSE at an earlier iteration 


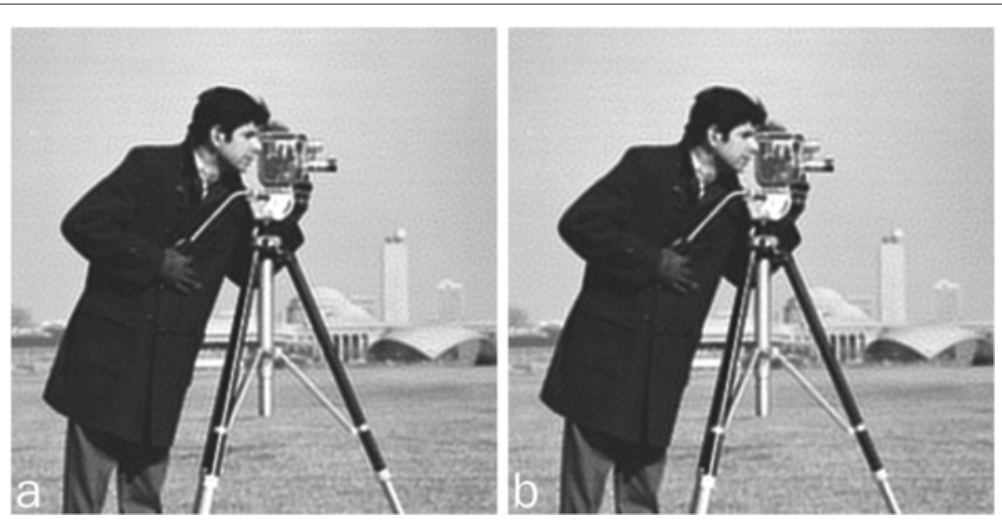

Fig. 7 Deblurred images by separated BI-RL a with $4 \times 4$ and $\mathbf{b} 16 \times 16$ rectangular blocks. In combing deblurred block images in $\mathbf{a}$ and $\mathbf{b}$, weights in (11) were used. These images were obtained at the 432th iteration with RSE $=0.53 \%$ for (a) and the 513 th iteration with RSE $=0.55 \%$ for (b)

in the Gaussian deblurring (the iteration is accelerated by about NB times).

Figure 9 shows deblurred images by interlaced BI-RL with $4 \times 4$ (a) and $8 \times 8$ (b) rectangularly-down-sampled blocks. Figure 9a was obtained at the 26th iteration with $\mathrm{RSE}=0.52 \%$ and Fig. $9 \mathrm{~b}$ at the second iteration with $\mathrm{RSE}=0.91 \%$. The comparison with Fig. 6a, obtained at the 420th iteration with RSE $=0.52 \%$ by RL, shows that interlaced BI-RL with $4 \times 4$ rectangularly-down-sampled blocks (Fig. 9a) maintains the deblurring quality comparable to RL, while interlaced BI-RL with $8 \times 8$ rectangularlydown-sampled blocks (Fig. 9b) produces severe ringing artifacts.

Results in Table 3 and Fig. 9 show that interlaced BI-RL with up to $4 \times 4$ rectangularly-down-sampled blocks achieves the desired goal (the accelerated iteration, with deblurring quality maintained) in the Gaussian deblurring.

Poor results by interlaced BI-RL with $8 \times 8$ rectangularly-down-sampled blocks for the Gaussian deblurring can be explained by the following argument. The performance of the $i$ th interlaced BI-RL sub-iteration (the step I4),

$$
\mathbf{f}^{m+i / t}=\left.\mathbf{f}^{m+(i-1) / t}\right|_{\Omega_{i}} * \frac{\mathcal{T}_{i}^{*} \mathbf{s}}{\mathcal{T}_{i}^{*} \mathbf{I}_{\Lambda_{i}}}, \quad \mathbf{s}=\frac{\mathbf{g}^{[i]}}{\mathcal{T}_{i}\left(\left.\mathbf{f}^{m+(i-1) / t}\right|_{\Omega_{i}}\right)},
$$

Table 2 Computation times for separated BI-RL with diagonal blocks for the diagonal deblurring and their smallest RSE results

\begin{tabular}{ccccccc}
\hline NB & BP & SB & CB & RSE(\%) & IN & PC \\
\hline 1 & n.a. & 0.658 & n.a. & 0.65 & 102 & 67 \\
2 & 2.2 & 0.327 & 0.0074 & 0.65 & 104 & 36 \\
4 & 2.2 & 0.163 & 0.0076 & 0.65 & 104 & 19 \\
8 & 2.2 & 0.082 & 0.0079 & 0.65 & 103 & 10 \\
16 & 2.2 & 0.040 & 0.0086 & 0.65 & 104 & 6 \\
\hline
\end{tabular}

The same abbreviations in Table 1 were used here. The row starting with ' 1 ' in NB column represents results by $R L$ highly depends on the denominator $\mathcal{T}^{*} \mathbf{I}_{\Lambda_{i}}$ (often called normalization term in emission tomography). As mentioned earlier in Section 3.2, $\left(\mathcal{T}^{*} \mathbf{I}_{\Lambda_{i}}\right)_{j_{1}, j_{2}}$ can be interpreted as the probability with which the pixel $\left(j_{1}, j_{2}\right) \in$ $\Omega_{i}$ contributes the observation on $\Lambda_{i}$. It is true that the value at the pixel $\left(j_{1}, j_{2}\right) \in \Omega_{i}$ with bigger $\left(\mathcal{T}^{*} \mathbf{I}_{\Lambda_{i}}\right)_{j_{1}, j_{2}}$ is often recovered faster or more accurately than the value at the pixel with smaller one. Thus non-uniform and small $\mathcal{T}^{*} \mathbf{I}_{\Lambda_{i}}$ often leads to slow convergence and a non-uniform deblurring effect in the $i$ th interlaced BI-RL sub-iteration.

Figure 10 shows $\mathcal{T}_{i}^{*} \mathbf{I}_{\Lambda_{i}}$, where $\Lambda_{i}$ are one of pixel subsets formed by $4 \times 4$ (a) and $8 \times 8$ (b) rectangularly-downsampled blocks and $\mathcal{T}_{i}$ is the blurring transform associated with the Gaussian PSF $\mathbf{k}_{G}$ and $\Lambda_{i}$. The argument in the preceding paragraph implies that more uniform and higher intensities in Fig. 10a than in 10b give the main reason why Fig. 9a, deblurred by interlaced BI-RL with denominators whose intensities look like Fig. 10a, is better than Fig. 9b, deblurred by interlaced BI-RL with denominators whose intensities look like Fig. 10b.

\subsubsection{Diagonally-down-sampled blocks for diagonal deblurring}

Table 4 shows the same data as Table 3 for diagonallydown-sampled blocks for the diagonal deblurring. Again, as in Table 3, the computation time for the one round of interlaced BI-RL iterations slightly increases as NB increases and interlaced BI-RL reaches its smallest RSE at an earlier iteration, with the acceleration rate of $\mathrm{NB}$ times.

Figure 11 shows deblurred images by interlaced BI-RL with 4 (a) and 8 (b) diagonally-down-sampled blocks, and their zoomed parts in (c) and (d). Figure 11a was obtained at the 26th iteration with RSE $=0.65 \%$ and Fig. $11 \mathrm{~b}$ at the 12th iteration with $\mathrm{RSE}=0.67 \%$. The comparison with Fig. 6b, obtained at the 102th iteration with RSE $=0.65 \%$ by RL, shows that interlaced BI-RL with 4 diagonallydown-sampled blocks (Fig. 11a) maintains the deblurring 


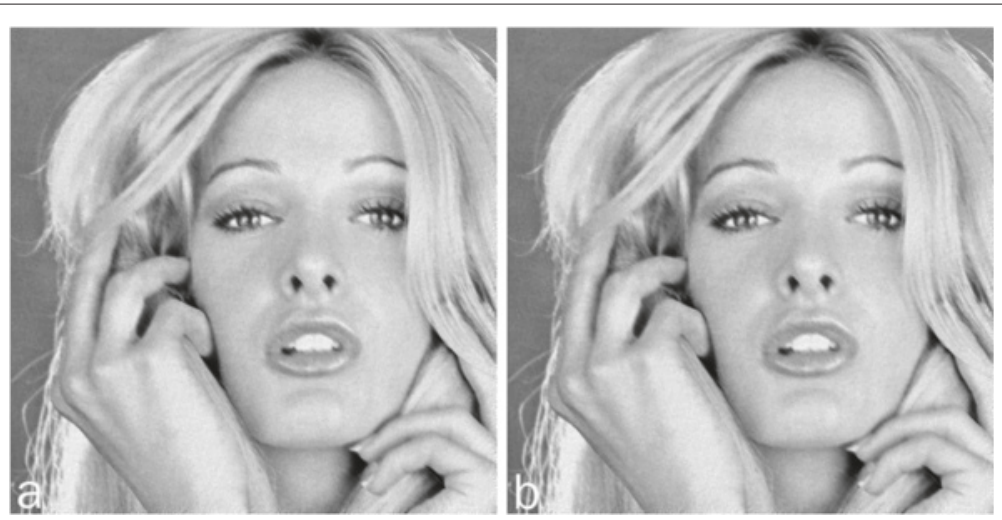

Fig. 8 Deblurred images by separated BI-RL with a 8 and $\mathbf{b} 16$ diagonal blocks. In combing deblurred block images, weights in (11) were used. These images were obtained at the 103th iteration with RSE $=0.65 \%$ for (a) and 104th iteration with RSE $=0.65 \%$ for $(\mathbf{b})$

quality comparable to RL, while interlaced BI-RL with 8 diagonally-down-sampled blocks exhibits some ringing artifacts (see zoomed parts Fig. 11c, d) with a slightly larger RSE.

Results in Table 4 and Fig. 11 show that interlaced BI$\mathrm{RL}$ with up to 6 diagonally-down-sampled blocks achieves its desired goal (the accelerated iteration, with deblurring quality maintained) in the diagonal deblurring.

Interlaced BI-RL with diagonally-down-sampled blocks for the diagonal deblurring did not accelerate the iteration as much as interlaced BI-RL with rectangularly-downsampled blocks did for the Gaussian deblurring. This phenomenon can be explained, again, by the uniformity and the largeness on denominators. Figure 12 shows $\mathcal{T}_{i}{ }^{*} \mathbf{I}_{\Lambda_{i}}$, where $\Lambda_{i}$ are one of pixel subsets formed by 4 (a) and 8 (b) diagonally-down-sampled blocks and $\mathcal{T}_{i}$ is the blurring transform associated with the diagonal PSF $\mathbf{k}_{D}$ and $\Lambda_{i}$. The uniformity comparison between Figs. 10a and 12a gives a partial reason why the diagonal deblurring is not easy to be accelerated as much as the Gaussian deblurring by interlaced BI-RL. It is also true that more uniform and higher intensities in Fig. 12a than in 12b makes Fig. 11a to be better than $11 \mathrm{~b}$.

Table 3 Computation times for interlaced BI-RL with rectangularly-down-sampled blocks for the Gaussian deblurring and their smallest RSE results

\begin{tabular}{cccccc}
\hline NB & BP & BI & RSE(\%) & IN & TC \\
\hline $1 \times 1$ & n.a. & 4.40 & 0.52 & 420 & 1,848 \\
$2 \times 2$ & 10.3 & 4.51 & 0.52 & 107 & 492 \\
$4 \times 4$ & 10.8 & 4.84 & 0.52 & 26 & 136 \\
$6 \times 6$ & 11.3 & 5.40 & 0.60 & 11 & 70 \\
$8 \times 8$ & 12.1 & 6.22 & 0.91 & 2 & 24
\end{tabular}

The abbreviation 'BI' represents the seconds spent for the one round of block iterations, while ' $T C^{\prime}$ ' shows the seconds spent for the total computation. The same abbreviations in Table 1 were used here

\subsection{Miscellaneous results}

Simulation studies described so far were repeated with Gaussian and diagonal PSFs of size $11 \times 11,35 \times 35$, and $51 \times 51$, different test images, different image sizes, and Gaussian noise models. We briefly report results of those simulation studies as follows.

- In Gaussian deblurrings, as the size of Gaussian PSF increased, separated BI-RL maintained the convergence rate of RL to smaller ranges of rectangular blocks.

- In diagonal deblurrings, separated BI-RL maintained the convergence rate of RL at least up to 16 diagonal blocks for all diagonal PSFs.

- In Gaussian deblurrings, as the size of Gaussian PSF increased, interlaced BI-RL accelerated iterations to wider ranges of rectangularly-down-sampled blocks.

- In diagonal deblurrings, as the size of diagonal PSF increased, interlaced BI-RL accelerated iterations to wider ranges of diagonally-down-sampled blocks.

- Smoother images had more chance of exhibiting block artifacts in separated BI-RL with rectangular blocks for Gaussian deblurrings. In separated BI-RL with diagonal blocks for diagonal deblurrings, the test image did not affect block artifacts.

- The use of Gaussian noise models itself did not affect the performance of proposed methods.

- Deblurring with noisier data had less chance of exhibiting block artifacts in separated BI-RL with rectangular blocks for Gaussian deblurrings. In separated BI-RL with diagonal blocks for diagonal deblurrings, the noise level did not affect block artifacts.

- Separated BI-RL worked better for larger sized images, while the performance of interlaced BI-RL did not depend on the image size. 


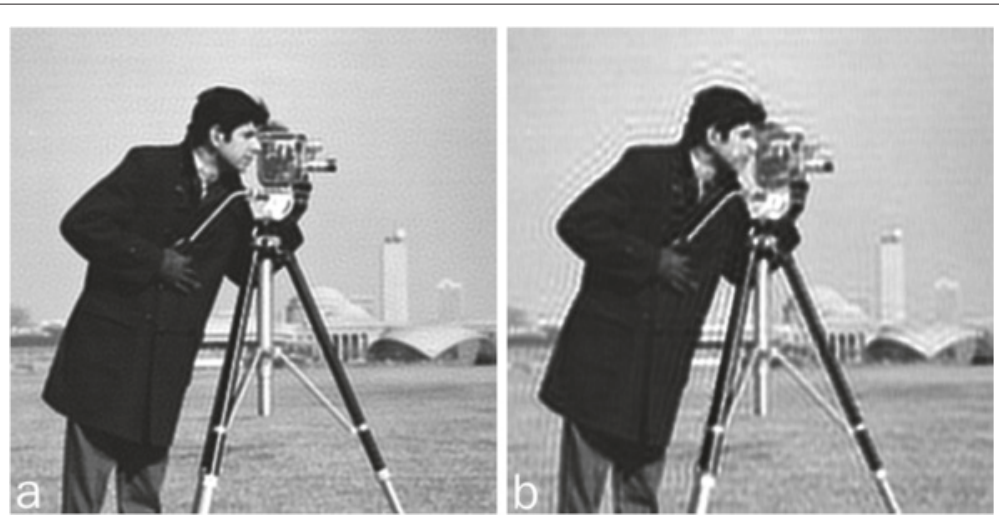

Fig. 9 Deblurred images by interlaced BI-RL with $\mathbf{a} 4 \times 4$ and $\mathbf{b} 8 \times 8$ rectangularly-down-sampled blocks. These images were obtained at the 26 th iteration with $\mathrm{RSE}=0.52 \%$ for $(\mathbf{a})$ and the second iteration with $\mathrm{RSE}=0.91 \%$ for $(\mathbf{b})$

We tested proposed methods with an image taken from a real camera. Figure 13a shows a blurred and noisy image taken by the famous photographer Robert Capa [30]. Figure 13b, c, d shows deblurred images by RL, separated BI-RL with 4 horizontal blocks, and interlaced BI-RL with 4 horizontally-down-sampled blocks, respectively. Here, horizontal blocks for separated BI-RL and horizontallydown-sampled blocks for separated BI-RL were selected by following two suggestions explained in Section 3.6 (the estimated PSF for Fig. 13a is known to be spread horizontally [30]).

Figure 13b, c shows 100th iterates of RL and separated BI-RL with 4 horizontal blocks, respectively. No noticeable difference between Fig. 13b, c indicates that separated BI-RL achieved its desired goal (the block artifact-free block deblurring) in this simulation. Figure 13d shows the 25th iterates of interlaced BI-RL with 4 horizontallydown-sampled blocks. Again, no noticeable difference between Fig. 13b, d indicates that interlaced BI-RL achieved its desired goal (the accelerated iteration) in this simulation.
We tested proposed methods with an image taken from a real camera. Figure 13a shows a blurred and noisy image (of size $316 \times 480$ ) taken by the famous photographer Robert Capa [30]. Figure 13b, c, d shows deblurred images by RL, separated BI-RL with 4 horizontal blocks, and interlaced BI-RL with 4 horizontally-down-sampled blocks, respectively. Here, horizontal blocks for separated BI-RL and horizontally-down-sampled blocks for separated BI-RL were selected by following two suggestions explained in Section 3.6 (the estimated PSF for Fig. 13a is known to be spread horizontally [30]; see Fig. 15a).

Figure 13b, c shows 100th iterates of RL and separated BI-RL with 4 horizontal blocks, respectively. No noticeable difference between Fig. 13b, c indicates that separated BI-RL achieved its desired goal (the block artifact-free block deblurring) in this simulation. Figure 13d shows the 25th iterates of interlaced BI-RL with 4 horizontallydown-sampled blocks. Again, no noticeable difference between Fig. 13b and 13d indicates that interlaced BI-RL achieved its desired goal (the accelerated iteration) in this simulation.
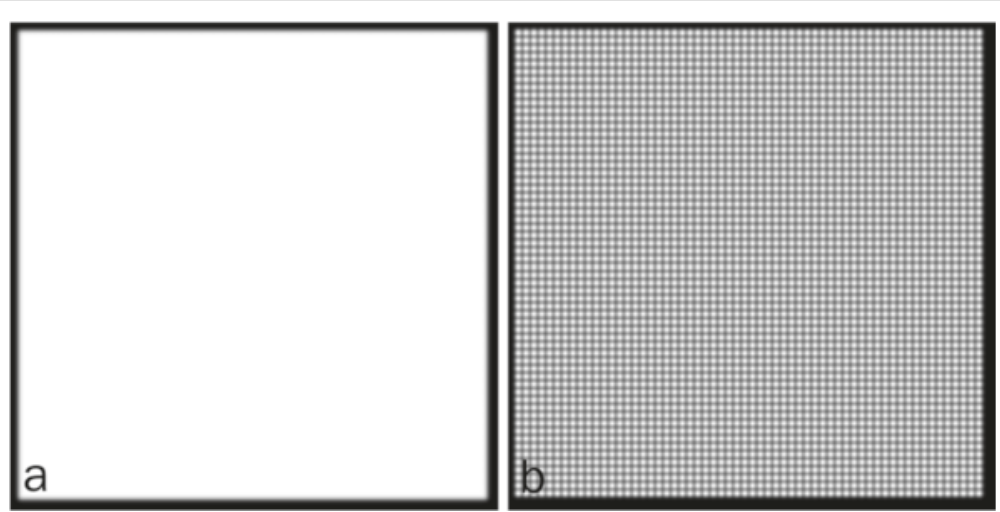

Fig. 10 Backprojected images $\boldsymbol{\mathcal { T }}_{i}^{*} \mathbf{I}_{\Lambda_{i}}$ of $\mathbf{a} 4 \times 4$ and $\mathbf{b} 8 \times 8$ rectangularly-down-sampled $\Lambda_{i}$. Here, $\boldsymbol{\mathcal { T }}_{i}$ is the blurring transform associated with the Gaussian PSF $\mathbf{k}_{G}$ and $\Lambda_{i}$ 
Table 4 Computation times for interlaced BI-RL with diagonally-down-sampled blocks for the diagonal deblurring and their smallest RSE results

\begin{tabular}{cccccc}
\hline NB & BP & BI & RSE(\%) & IN & TC \\
\hline 1 & n.a. & 0.65 & 0.65 & 102 & 66 \\
2 & 2.2 & 0.69 & 0.65 & 52 & 38 \\
4 & 2.3 & 0.75 & 0.65 & 26 & 21 \\
6 & 2.3 & 0.81 & 0.65 & 17 & 16 \\
8 & 2.4 & 0.88 & 0.67 & 12 & 12 \\
\hline
\end{tabular}

Here, same abbreviations and conventions in Tables 1 and 3 were used

We also tested proposed methods with a color image taken from a real camera. Figure 14 a shows a color 'summer house' image of size $946 \times 952$ in [30]. Figure 14b, c shows 100th iterates of RL and separated BI-RL with $2 \times 2$ rectangular blocks, respectively. No noticeable difference between Fig. 14b, c indicates that separated BI-RL achieved its desired goal (the block artifact-free block deblurring) in this simulation. Figure $14 \mathrm{~d}$ shows the 25 th iterates of interlaced BI-RL with $2 \times 2$ rectangularly-downsampled blocks. Again, no noticeable difference between Fig. 14b, d indicates that interlaced BI-RL achieved its desired goal (the accelerated iteration) in this simulation.
Figure 15 shows PSF images that were estimated from observed images, Figs. 13a for 15a and Figs. 14a for 15b, in [30].

We also conducted simulation studies on separated BI-RL with 'improperly chosen blocks.' Figure 16 shows deblurred images by separated BI-RL with diagonal blocks for the Gaussian deblurring (a) and rectangular blocks for the diagonal deblurring (b). In combining deblurred block images, weights $\mathbf{r}^{[i]}, i=$ $1, \ldots, t$, in (11) were used. Figure 16a did not show block artifacts, while Fig. 16b suffered from block artifacts.

Block artifacts in Fig. 16b were caused by boundary artifacts generated in diagonal deblurrings of rectangular blocks. In fact, the Gaussian deblurring also generated boundary artifacts for both rectangular and diagonal blocks. Those boundary artifacts were, however, confined only in outermost part of $\Omega_{i}-\Lambda_{i}$ and did not appear in the final image (Fig. 16a), since $\mathbf{r}^{[i]}$ in (11) were very small for image pixels $\left(j_{1}, j_{2}\right) \in \Omega_{i}-\Lambda_{i}$ where boundary artifacts were strong. In diagonal deblurrings of rectangular blocks, however, boundary artifacts appeared in the final image (Fig. 16b) because of the exact opposite reason. The asymmetrical and slow decay of the diagonal PSF $\mathbf{k}_{D}$ makes such difference.

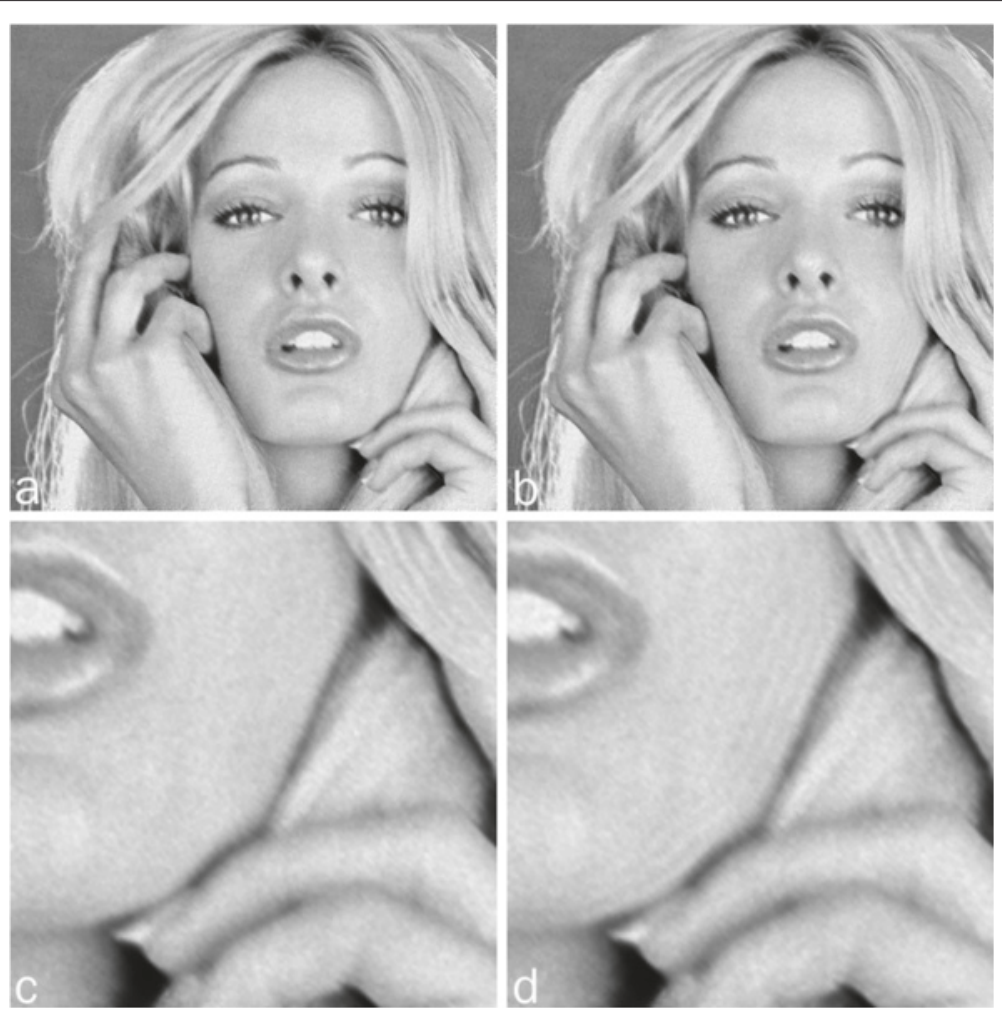

Fig. 11 Deblurred images by interlaced BI-RL with diagonally-down-sampled blocks and their zoomed parts. a 4 and b 8 diagonally-down-sampled blocks, c a zoomed part of (a) and $\mathbf{d}$ a zoomed part of $(\mathbf{b})$. These images were obtained at the 26th iteration with RSE $=0.65 \%$ for $(\mathbf{a})$ and 12 th iteration with $\mathrm{RSE}=0.67 \%$ for $(\mathbf{b})$ 


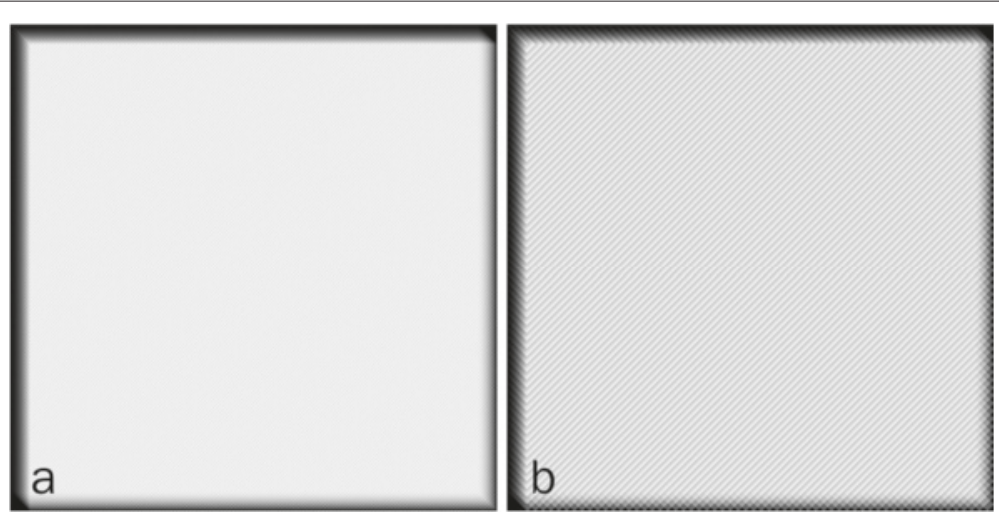

Fig. 12 Backprojected images $\mathcal{T}_{i}^{*} \mathbf{I}_{\Lambda_{i}}$ of $\mathbf{a} 4$ and $\mathbf{b} 8$ diagonally-down-sampled $\Lambda_{i}$. Here, $\boldsymbol{T}_{i}$ is the blurring transform associated with the diagonal PSF $\mathbf{k}_{D}$ and $\Lambda_{i}$

On the other hand, diagonal deblurrings of diagonal blocks do not produce block artifacts, as shown in Fig. 8; most of boundary artifacts from diagonal deblurrings of diagonal blocks appear only in $\Omega-\Lambda$ and hence can be easily removed by cutting out pixels in $\Omega-\Lambda$. These results show that, in case when the formula (11) is used for the combining of deblurred block images, the shape of blocks is important for the diagonal deblurring but not for the Gaussian deblurring.

Selecting blocks depending on the PSF is not an easy task. To deal with this problem, we suggest to use overlapped rectangular blocks $\Lambda_{i}$ for separated BI-RL, in the case when it is not certain which blocks should be chosen.

Figure 17 shows deblurred images by separated BI-RL with overlapped rectangular blocks for Gaussian (a) and diagonal (b) deblurrings. To be specific, Fig. 17a shows the deblurred image by separated BI-RL with $16 \times 16$ overlapped rectangular blocks. These overlapped blocks were formed from $16 \times 16$ disjoint rectangular blocks (the size of each block is $30 \times 30$ ) by adding nine rows or nine columns to boundaries of blocks.

Separated BI-RL with these overlapped rectangular blocks produces 256 deblurred block images and combines 256 deblurred block images of size $30 \times 30$, after cutting out required amount of rows and columns from 256 deblurred block images, to the final image (Fig. 17a). Figure 17b can be obtained by a similar procedure.

Both images in Fig. 17 do not show block artifacts (see Fig. 18, which shows zoomed parts of Figs. 16b and 17b, respectively, for a better comparison). These results indicate that overlapped rectangular blocks can be used for

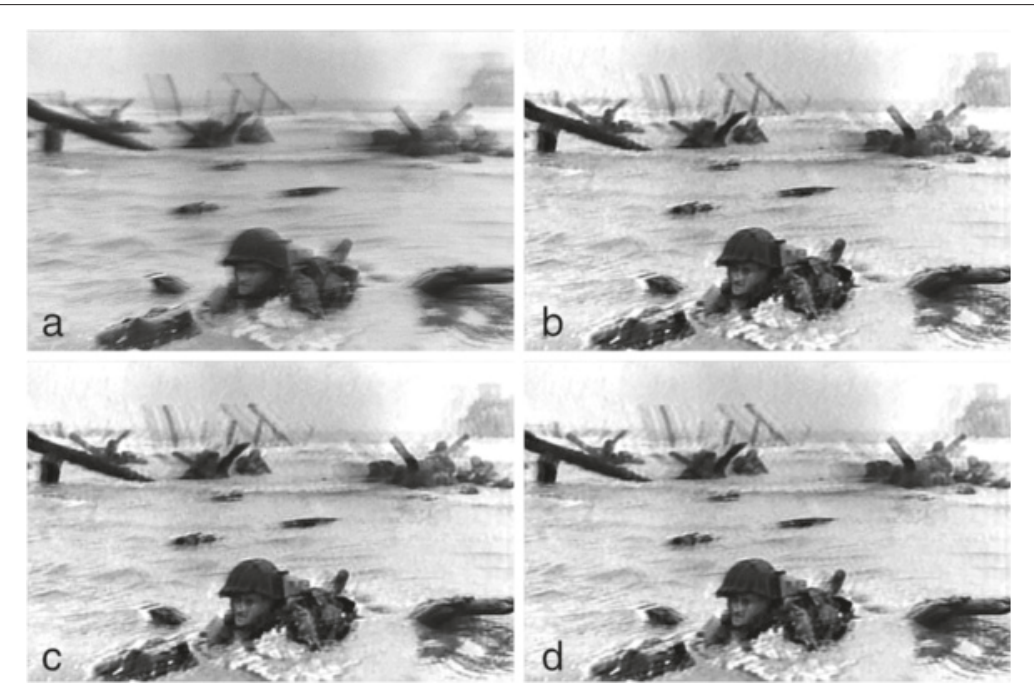

Fig. 13 Test of proposed methods with an image taken from a real camera. a The image (of size 316 × 480) taken by Robert Capa on 6 June 1944 [30], $\mathbf{b}$ the deblurred image by RL, $\mathbf{c}$ the deblurred image by separated BI-RL with 4 horizontal blocks, and $\mathbf{d}$ the deblurred image by interlaced BI-RL with 4 horizontally-down-sampled blocks. Here, 100, 100, and 25 were used as iteration numbers for (b), (c), and (d), respectively 


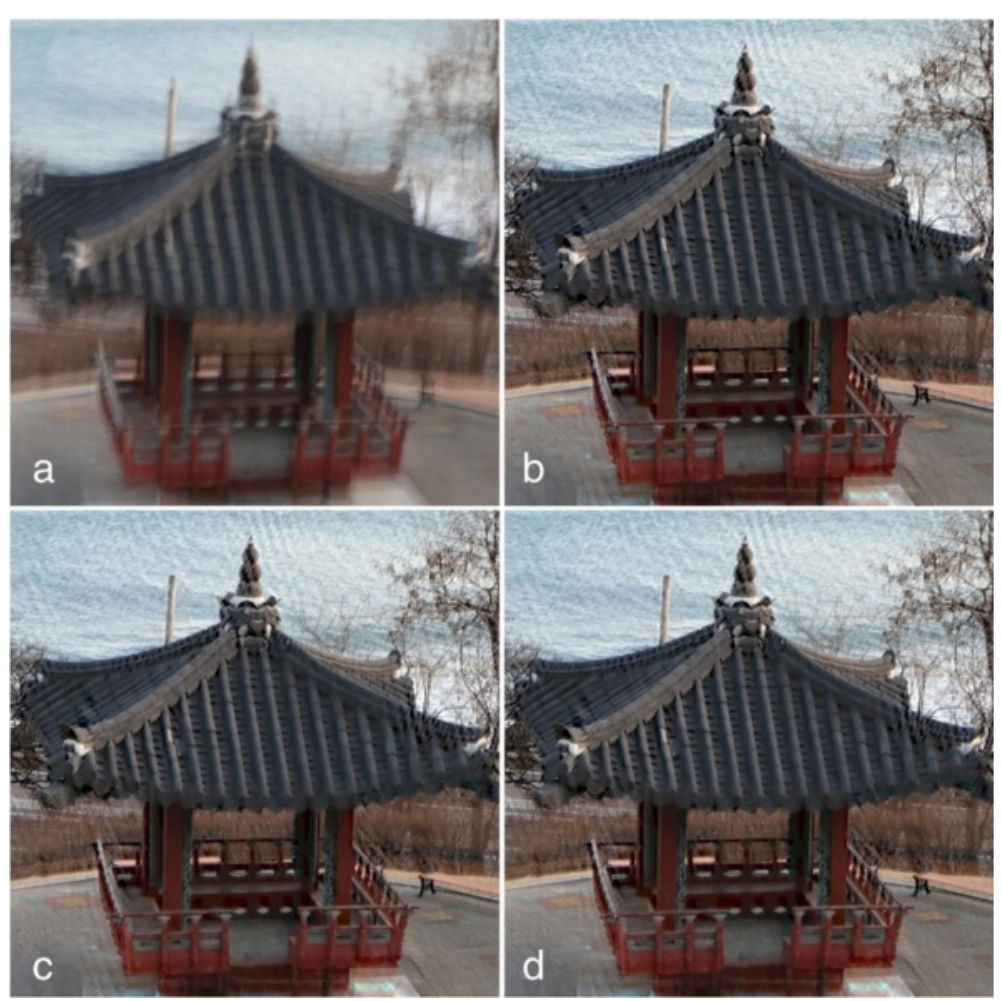

Fig. 14 Test of proposed methods with a color image taken from a real camera. a A color 'summer house' image (of size $946 \times 952$ ) taken from a real camera [30], $\mathbf{b}$ the deblurred image by RL, $\mathbf{c}$ the deblurred image by separated BI-RL with $2 \times 2$ rectangular blocks, and $\mathbf{d}$ the deblurred image by interlaced BI-RL with $2 \times 2$ rectangularly-down-sampled blocks. Here, 100, 100, and 25 were used as iteration numbers for (b), (c), and (d), respectively

separated BI-RL for any kind of PSFs, at the cost of additional computations caused by overlapped pixels; for the Gaussian deblurring with $4 \times 4$ rectangular blocks, disjoint blocks took $0.275 \mathrm{~s}$ for the iteration for the one block, while $4 \times 4$ overlapped rectangular blocks formed by adding nine pixel rows and columns to boundaries took $0.337 \mathrm{~s}$
As mentioned in Section 2, interlaced BI-RL was compared with the acceleration technique in [7]. Figure 19a shows the result of the acceleration technique in [7], which obtained the smallest RSE $0.54 \%$ at the 41 st iteration in $182.4 \mathrm{~s}(=4.45 \mathrm{~s} \times 41)$. On the other hand, Fig. 9a, obtained at the 26th iteration with RSE $0.52 \%$ by interlaced BI-RL with $4 \times 4$ rectangularly-down-sampled

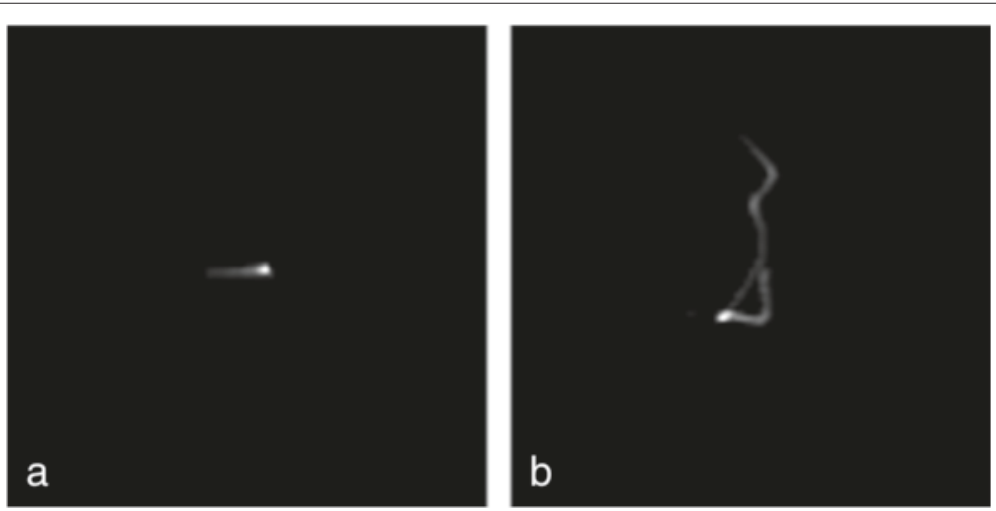

Fig. 15 Estimated PSFs. a The PSF that was estimated from the observed image in Fig. 13a in [30]. Figure 13b, c, d was computed by using this PSF b The PSF that was estimated from the observed image in Fig. 14a in [30]. Figure 14b, c, d was computed by using this PSF. Here, both PSF were displayed as images of size $101 \times 101$ 


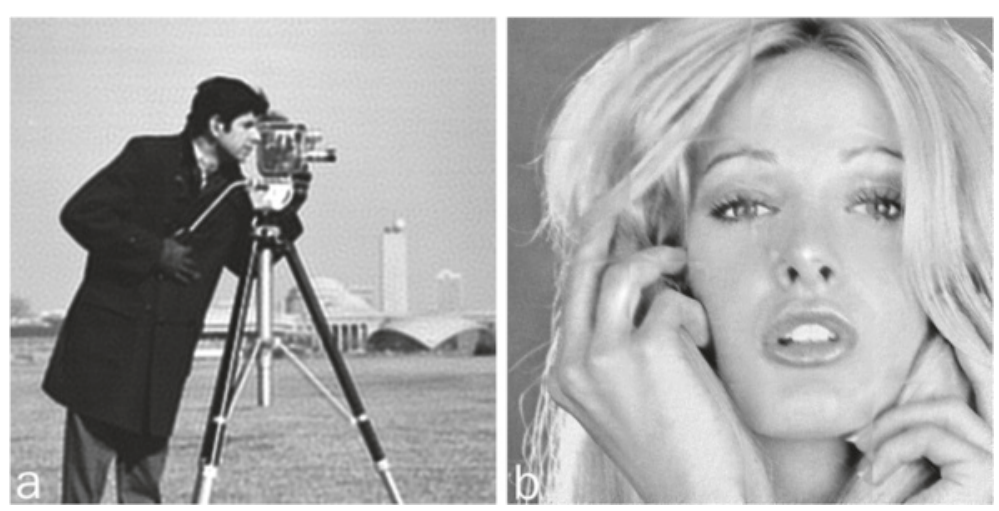

Fig. 16 Deblurred images by separated BI-RL with improperly chosen blocks. In combing deblurred block images, the formula (11) was used. a Diagonal blocks for the Gaussian deblurring and $\mathbf{b}$ rectangular blocks for the diagonal deblurring

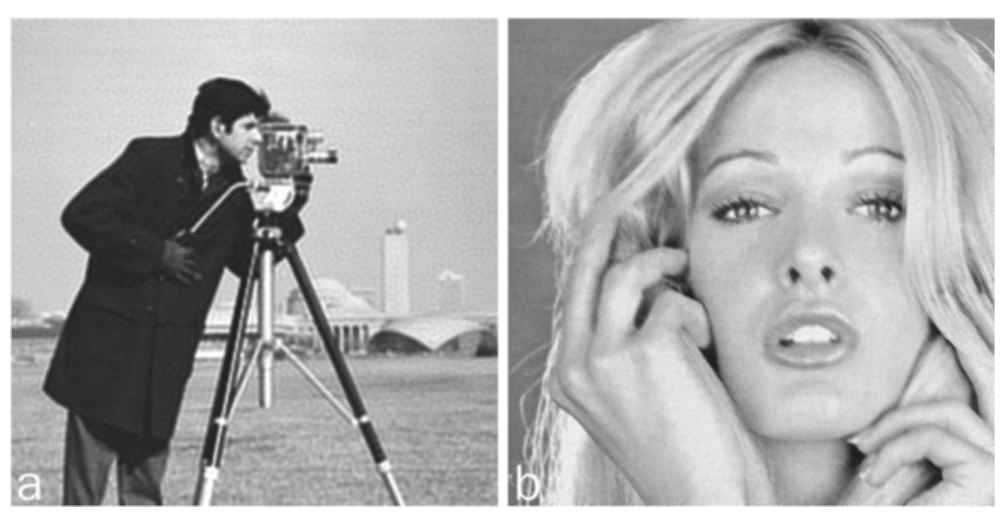

Fig. 17 Deblurred images by separated BI-RL with overlapped rectangular blocks. a Gaussian and $\mathbf{b}$ diagonal deblurrings

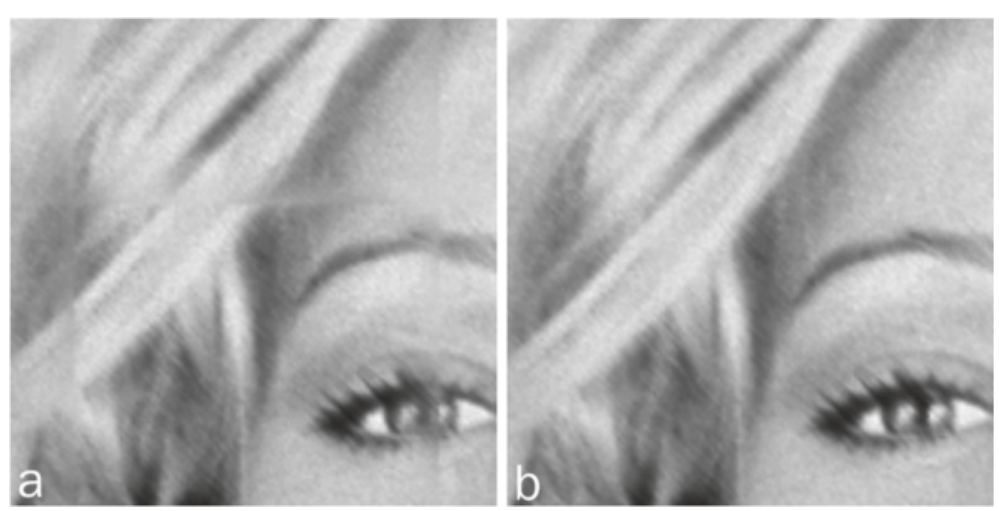

Fig. 18 Zoomed parts. a A zoomed part of Fig. 16b and $\mathbf{b}$ a zoomed part of Fig. 17b 


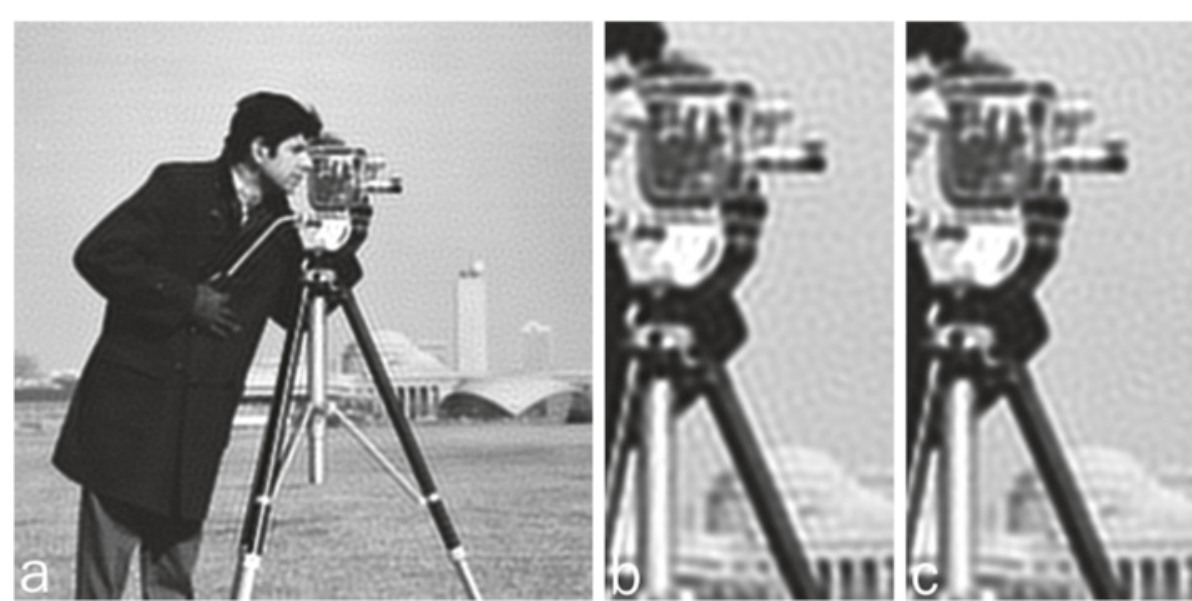

Fig. 19 Acceleration comparison. a Deblurred image by the accelerated RL method in [7], obtained at the 41st iteration with RSE $=0.54 \%$, b a zoomed part of (a), and $\mathbf{c}$ a zoomed part of Fig. 9a, obtained by interlaced BI-RL with $4 \times 4$ rectangularly-down-sampled blocks at the 26 th iteration with RSE $=0.52 \%$

blocks, took $136.6 \mathrm{~s}(10.81 \mathrm{~s}$ for the block partition + $4.84 \mathrm{~s} \times 26$ for iterations). The comparison of Fig. 19a with Fig. 9a shows that the technique in [7] provided a slightly slower acceleration and larger RSE than interlaced BI-RL with $4 \times 4$ rectangularly-down-sampled blocks. It also shows that the technique in [7] produced slightly more ringing artifacts and noise amplification than interlaced BI-RL. For a better visual comparison, see Fig. 19b, c, which shows zoomed parts of Figs. 19a and 9a, respectively.

\section{Conclusions}

In this paper, we extended RL to block-iterative versions, separated BI-RL, and interlaced BI-RL, for image deblurring applications. We conducted simulation studies to test proposed methods in Gaussian and diagonal deblurrings. Simulation results showed that separated BI-RL can have a benefit of parallel computations for Gaussian and diagonal deblurrings, with a wide range of rectangular and diagonal blocks, respectively. Simulation results also showed that interlaced BI-RL can accelerate the iteration for Gaussian and diagonal deblurrings but only with a limited range of rectangularly- and diagonally-down-sampled blocks, respectively.

In this work, proposed methods were tested only for Gaussian and diagonal PSFs, $\mathbf{k}_{G}$ and $\mathbf{k}_{D}$ in Fig. 3. It is clear that proposed methods can be extended to other PSFs as long as admissible blocks can be selected. But, as mentioned earlier, it is not clear how blocks should be formed for a specific PSF. Simulation results in Section 4 indicates that overlapped rectangular blocks can be used as universal admissible blocks for separated BI-RL, at the cost of additional computations caused by overlapped pixels. For interlaced BI-RL, however, such universal admissible blocks are not known yet.

\section{Competing interests}

The author declares that he has no competing interests.

\section{Acknowledgements}

This work was supported by the Inje Research and Scholarship Foundation in 2012 and conducted while the author was a visiting scholar at the Center for Nonlinear Analysis, Department of Mathematical Sciences, Carnegie Mellon University. The author would like to express his sincere appreciation to Prof. Lucier at Purdue University for his helpful suggestions.

Received: 30 August 2013 Accepted: 9 May 2015

Published online: 04 June 2015

\section{References}

1. AK Jain, Fundamentals of Digital Image Processing. (Prentice-Hall, Englewood Cliffs, NJ, 1989)

2. W Richardson, Bayesian-based iterative method of image restoration. J. Opt. Soc. Am. 62(1), 55-59 (1972)

3. L Lucy, An iterative techniques for the rectification of observed distributions. Astronomical J. 79(6), 745-754 (1974)

4. M Bertero, P Boccacci, G Desiderá, G Vicidomini, Image deblurring with Poisson data: from cells to galaxies. Inverse Probl. 25, 123006 (2009)

5. Y-W Tai, P Tan, M Brown, Richardson-Lucy deblurring for scenes under a projective motion path. IEEE Trans. PAMI. 33(8), 1603-1618 (2011)

6. H Hudson, R Larkin, Accelerated image reconstruction using ordered subsets of projection data. IEEE Trans. Med. Imaging. 13(4), 601-609 (1994)

7. D Biggs, $M$ Andrews, Acceleration of iterative image restoration algorithms. Appl. Optics. 36(8), 1766-1775 (1997)

8. L Yuan, J Sun, L Quan, H Shum, Progressive inter-scale and intra-scale non-blind image deconvolution. ACM Trans. Graph. 27(3), 74-17410 (2008)

9. S Setzer, G Steidl, T Teuber, Deblurring Poissonian images by split Bregman techniques. J. Vis. Commun. Image Represent. 21(3), 193-199 (2010)

10. Y Wang, H Feng, Z Xu, Q Li, C Dai, An improved Richardson-Lucy algorithm based on local prior. Opt. Laser Technol. 42(5), 845-849 (2010)

11. M Temerinac-Ott, Tile-based Lucy-Richardson deconvolution modeling a spatially-varying PSF for fast multiview fusion of microscopical images. (Technical report 260, University of Freiburg, 2010). http://Imb.informatik. uni-freiburg.de//Publications/2010/Tem10a

12. J-L Wu, C-F Chang, C-S Chen, An adaptive Richardson-Lucy algorithm for single image deblurring using local extrema filtering. J. Appl. Sci. Eng. 16(3), 269-276 (2013)

13. L Shepp, Y Vardi, Maximum likelihood reconstruction for emission tomography. IEEE Trans. Med. Imaging. 1(2), 113-122 (1982) 
14. A Dempster, N Laird, D Rubin, Maximum likelihood from incomplete data via the EM algorithm. J. R. Stat. Soc. Series B. 39(1), 1-38 (1977)

15. N-Y Lee, Y Choi, A modified OSEM algorithm for pet reconstruction using wavelet processing. Comput. Methods Prog. Biomed. 80(3), 236-245 (2005)

16. A Reilhaca, S Tomeïa, I Buvatb, C Michel, F Keherenc, N Costesa, Simulation-based evaluation of OSEM iterative reconstruction methods in dynamic brain pet studies. Neurolmage. 39(1), 359-368 (2008)

17. M Bertero, P Boccacci, A simple method for the reduction of boundary effects in the Richardson-Lucy approach to image deconvolution. Astron. Astrophys. 437, 369-374 (2005)

18. B Anconelli, M Bertero, P Boccacci, G Desiderá, M Carbillet, H Lanteri, Deconvolution of multiple images with high dynamic range and an application to LBT LINC-NIRVANA. Astron. Astrophys. 460, 349-355 (2006)

19. H Adorf, R Hook, L Lucy, F Murtagh, Accelerating the Richardson-Lucy restoration algorithm. Proc. 4th ESO/ST-ECF Data Analysis Workshop, European Southern Observatory, 99-103 (1992)

20. THolmes, Y Liu, Acceleration of maximum-likelihood image restoration for fluorescence microscopy and other noncoherent imagery. J. Opt. Soc. Am. A. 8(6), 893-907 (1991)

21. L Kaufman, Implementing and accelerating the EM algorithm for positron emission tomography. IEEE Trans. Med. Imaging. 6(1), 37-51 (1997)

22. A Tekalp, M Sezan, Quantitative analysis of artifacts in linear space-invariant image restoration. Multidimensional Syst. Signal Process. 1, 143-177 (1990)

23. $\mathrm{M} \mathrm{Ng}, \mathrm{R}$ Chan, W-C Tang, A fast algorithm for deblurring models with Neumann boundary conditions. SIAM J. Sci. Comput. 21(3), 851-866 (1990)

24. S Serra-Capizzano, A note on anti-reflective boundary conditions and fast deblurring models. SIAM J. Sci. Comput. 25(4), 1307-1325 (2003)

25. M Donatelli, S Serra-Capizzano, Anti-reflective boundary conditions and re-blurring. Inverse Probl. 21(1), 169-182 (2005)

26. M Donatelli, C Estatico, A Martinelli, S Serra-Capizzano, Improved image deblurring with anti-reflective boundary conditions and re-blurring. Inverse Probl. 22(6), 2035-2053 (2006)

27. R Liu, J Jia, Reducing boundary artifacts in image deconvolution. IEEE Int. Conf. Image Process. - ICIP., 505-508 (2008)

28. D Calvetti, J Kaipio, E Somersalo, Aristotelian prior boundary conditions. Int. J. Math. Comput. Sci. 1, 63-81 (2006)

29. N-Y Lee, B Lucier, Preconditioned conjugate gradient method for boundary artifact-free image deblurring. Technical report, Carnegie Mellon University, Department of Mathematical Sciences (2013). http://www.math.cmu.edu/CNA/Publications/publications2013/011abs/ 13-CNA-011.pdf

30. Deblur Famous/Interesting Photos. http://www.juew.org/ deblurFamousPhoto.html

\section{Submit your manuscript to a SpringerOpen ${ }^{\circ}$ journal and benefit from:}

- Convenient online submission

- Rigorous peer review

- Immediate publication on acceptance

- Open access: articles freely available online

- High visibility within the field

- Retaining the copyright to your article

Submit your next manuscript at $\boldsymbol{\triangleright}$ springeropen.com 